\title{
Bifurcating action of Smoothened in Hedgehog signaling is mediated by Dlg5
}

\author{
Yong Chun Chong, ${ }^{1,2,3}$ Randall K. Mann, ${ }^{1,2,3}$ Chen Zhao, ${ }^{1,2,3}$ Masaki Kato, ${ }^{1,2}$ \\ and Philip A. Beachy ${ }^{1,2,3,4}$ \\ ${ }^{1}$ Institute for Stem Cell Biology and Regenerative Medicine, Stanford University, Stanford, California 94305, USA; ${ }^{2}$ Department \\ of Developmental Biology, Stanford University, Stanford, California 94305, USA; ${ }^{3}$ Department of Biochemistry, Stanford \\ University, Stanford, California 94305, USA; ${ }^{4}$ Howard Hughes Medical Institute, Stanford University, Stanford, California \\ 94305, USA
}

Binding of the Hedgehog $(\mathrm{Hh})$ protein signal to its receptor, Patched, induces accumulation of the seven-pass transmembrane protein Smoothened (Smo) within the primary cilium and of the zinc finger transcription factor Gli2 at the ciliary tip, resulting ultimately in Gli-mediated changes in nuclear gene expression. However, the mechanism by which pathway activation is communicated from Smo to Gli2 is not known. In an effort to elucidate this mechanism, we identified Dlg5 (Discs large, homolog 5) in a biochemical screen for proteins that preferentially interact with activated Smo. We found that disruption of Smo-Dlg5 interactions or depletion of endogenous Dlg5 leads to diminished Hh pathway response without a significant impact on Smo ciliary accumulation. We also found that Dlg5 is localized at the basal body, where it associates with another pathway component, Kif7. We show that Dlg5 is required for Hh-induced enrichment of Kif7 and Gli2 at the tip of the cilium but is dispensable for Gpr161 exit from the cilium and the consequent suppression of Gli3 processing into its repressor form. Our findings suggest a bifurcation of Smo activity in Hh response, with a Dlg5-independent arm for suppression of Gli repressor formation and a second arm involving Smo interaction with Dlg5 for Gli activation.

[Keywords: basal body; Dlg5; Gli; Hedgehog; Kif7; Smoothened]

Supplemental material is available for this article.

Received September 13, 2014; revised version accepted December 29, 2014.

The Hedgehog (Hh) signaling pathway specifies embryonic pattern and functions post-embryonically in tissue regeneration and homeostasis (Beachy et al. 2004; Varjosalo and Taipale 2008; Ingham et al. 2011). Inappropriate activation of the Hh pathway has been linked to malignancies such as basal cell carcinoma and medulloblastoma (Teglund and Toftgard 2010). Hh signaling is initiated by the binding of Hh ligand to its receptor, the 12-pass transmembrane protein Patched (Ptch), thus relieving Ptch-mediated inhibition of the seven-pass transmembrane protein Smoothened (Smo). Smo then accumulates in the primary cilium, a nonmotile microtubule-based projection from the cell surface (Corbit et al. 2005; Rohatgi et al. 2007), and this is accompanied by the ciliary exit of the GPCR Gpr161 and a concomitant reduction in basal cAMP levels in the cilium (Mukhopadhyay et al. 2013). Canonical signaling results in the suppression of Gli processing into a truncated repressor, the accumulation of Gli proteins at the tip of the cilium, and, ultimately, Gli activation and translocation to the nucleus, where Hh target genes such as Gli1 are transcriptionally activated (Chen et al. 2009; Kim et al. 2009).

Corresponding author: pbeachy@stanford.edu

Article is online at http://www.genesdev.org/cgi/doi/10.1101/gad.252676.114.
Our understanding of the Hh pathway is incomplete, due in part to the fact that even though core components of the pathway are essentially conserved, significant mechanistic differences exist between Drosophila and vertebrates. Specifically, a cytoplasmic complex comprising the kinase Fused $(\mathrm{Fu})$ and the atypical kinesin Costal-2 (Cos2) that links activated Smo and the Gli ortholog Ci in Drosophila does not seem to play a similar role in vertebrates (Robbins et al. 1997; Stegman et al. 2000; Lum et al. 2003). Thus, $\mathrm{Fu}^{-/-}$mice do not exhibit phenotypes characteristic of abnormal $\mathrm{Hh}$ activity; instead, $\mathrm{Fu}$ is required for the formation of motile $9+2$ cilia (Merchant et al. 2005; Wilson et al. 2009). The Kif7 ortholog of Cos2, on the other hand, is genetically implicated in $\mathrm{Hh}$ signaling, binds the Sufu/Gli complex, and is involved in the proteolytic processing of Gli into a transcriptional repressor (Cheung et al. 2009; Endoh-Yamagami et al. 2009; Liem et al. 2009; Maurya et al. 2013), but the role of

(C) 2015 Chong et al. This article is distributed exclusively by Cold Spring Harbor Laboratory Press for the first six months after the full-issue publication date (see http://genesdev.cshlp.org/site/misc/terms.xhtml). After six months, it is available under a Creative Commons License (Attribution-NonCommercial 4.0 International), as described at http:// creativecommons.org/licenses/by-nc/4.0/. 
Kif7 or other proteins in linking activated Smo and Gli in the primary cilium remains unresolved. Several groups have attempted to fill this gap in understanding by performing genome-wide siRNA screens to identify novel components of the Hh signaling pathway (Evangelista et al. 2008; Hillman et al. 2011; Jacob et al. 2011). Unfortunately, lack of reliability in the efficacy and specificity of siRNAs coupled with the likelihood of such screens leading to the identification of targets that disrupt the integrity of the primary cilia rather than selectively act on the Hh pathway has complicated these efforts. Conversely, mass spectrometry (MS)-based methods have been used to successfully identify components of multiprotein complexes, including the aforementioned Smo/ Cos2/Fu/Ci complex in Drosophila (Lum et al. 2003).

Here, using GFP-tagged Smo (LAP-Smo) and stable isotope labeling for quantitative, MS-based measurement of relative protein abundances (Ong et al. 2002; Zhu et al. 2002; Hubner et al. 2010), we identify Dlg5 (encoded by Discs large, homolog 5) as a preferential binding partner of Smo upon Hh pathway activation. Dlg5 is a $240-\mathrm{kDa}$ cytoplasmic protein belonging to the membrane-associated guanylate kinase (MAGUK) superfamily of proteins and is homologous to a two-member family of Drosophila genes that includes the tumor suppressor $d l g$ and $d l g 5$ (Nakamura et al. 1998; Purmonen et al. 2002; Shah et al. 2002). Functionally, the murine Dlg5 has been reported to be essential for the maintenance of cellular apical-basal polarity (Nechiporuk et al. 2007). We show that loss of murine Dlg5 diminishes the cellular response to Sonic hedgehog (Shh), although it is not required for formation of the primary cilium or ciliary accumulation of Smo. The Dlg5 protein is not enriched in the cilium proper but instead localizes to the basal body at the base of the cilium, where we also found Kif7 to be enriched. Although loss of Dlg5 impairs Hh-induced Gli2 and Kif7 accumulation at the ciliary tip and subsequent translocation of Gli2 into the nucleus, we observed no defect in the Hh-induced exit of Gpr161 from the cilium or in Hh-dependent suppression of the proteolytic processing of Gli3 into a repressor. These data indicate that $\mathrm{Dlg} 5$ interaction with Smo is specifically required for the activation of Gli but not for Smo-induced ciliary exit of Gpr161 and consequent suppression of Gli repressor formation. Smo activity thus bifurcates, with Dlg5 function restricted to the arm of the pathway that functions to activate Gli.

\section{Results}

\section{Dlg5 is a Smo-interacting protein}

We generated several clones of $\mathrm{Smo}^{-1-}$ mouse embryonic fibroblasts (MEFs) that stably express LAP-Smo-a GFPtagged, fully functional form of Smo-and selected one (subsequently referred to as LAP-Smo MEF) that exhibited optimal response to the Hh pathway (Fig. 1A,B). Treatment of LAP-Smo MEF with ShhN conditioned medium or 200 nM SAG1.5 (Smo agonist 1.5) resulted in a 10-fold increase in Gli-driven luciferase reporter, and this activity was suppressed by coincubation with $3 \mu \mathrm{M}$ Smo antagonist cyclopamine (Fig. 1A). In addition, ShhN induced the accumulation of LAP-Smo in the primary cilium (Fig. $1 \mathrm{~B})$, indicating that the level of LAP-Smo expression in this cell line provided a normal response to activation and inhibition of the Hh pathway.

To identify proteins that preferentially interact with activated LAP-Smo, we made use of SILAC (stable isotope labeling with amino acids in cell culture), a labeling technique that permits MS-based identification of proteins selectively enriched in differently treated cell populations. In our experiment, populations of cells were grown in medium containing either "light" (i.e., naturally occurring) L-lysine and L-arginine or the "heavy" stable isotope analogs ${ }^{13} \mathrm{C}_{6},{ }_{15} \mathrm{~N}_{4}$-L-arginine and ${ }^{13} \mathrm{C}_{6}{ }^{15} \mathrm{~N}_{2}$ - $\mathrm{L}$ lysine. The "light" cells were stimulated with a combination of ShhNp and SAG1.5 to induce maximal activation of the Hh pathway, whereas the "heavy" population was left untreated. Following immunoprecipitation of Smo (and associated proteins) using an anti-GFP matrix, peptides derived from both populations were combined and analyzed via capillary liquid chromatography-coupled tandem MS (LC-MS/MS) (Fig. 1C,D). Among candidate Smo-interacting proteins that were enriched in the "light" population, we selected six of the top-ranked proteins that possess well-defined protein-protein interaction domains for subsequent validation (i.e., to confirm a specific association with Smo). To do so, we coexpressed LAP-Smo and individual 3xFlag-tagged candidate interactors in HEK293T cells and tested for coimmunoprecipitation. Three of these proteins-Epb4.115, drebrin 1 (Dbn1), and LYRIC—-did not exhibit specific binding to LAP-Smo, and expression of a fourth, kinectin (Ktn1), was not detectable. Significantly, syntenin-1 and both fulllength $\mathrm{Dlg} 5$ and its guanylate kinase-like (GK) domain (Dlg5-GK) interacted specifically with LAP-Smo (Fig. 1E; Supplemental Fig. S1). In this study, we focused on the functional implication of the Smo/Dlg5 interaction in the context of Hh signaling.

Our SILAC/MS experiment suggested that Shh/SAG1.5 treatment significantly increased Dlg 5 binding to Smo (Fig. 1D). To validate this effect of Shh stimulation, we made use of a second clonal LAP-Smo cell line that expresses LAPtagged Smo at lower levels (LAP-Smo2). We found that similar levels of Gli-driven luciferase activity (data not shown) and Smo ciliary accumulation (Supplemental Fig. S2) were induced by Shh in both cell lines. We found that LAP-Smo2 cells stimulated with ShhN for increasing periods of time and subsequently incubated with $1 \mathrm{mM}$ DSP (Lomant's reagent) cross-linker displayed a gradual increase in the level of Smo-Dlg5 interaction (Fig. 1F), confirming that $\mathrm{Hh}$ pathway activation promotes the association of Smo with Dlg5.

\section{Mapping the Smo-Dlg5 interaction motifs}

Dlg5 contains at least six motifs known to mediate proteinprotein interactions: four PDZ (postsynaptic density 95, discs large, zona occludens 1) domains followed by an SH3 (Src homology 3) domain and a GK domain that, like those of other MAGUK family proteins, is considered to be 
Chong et al.

A

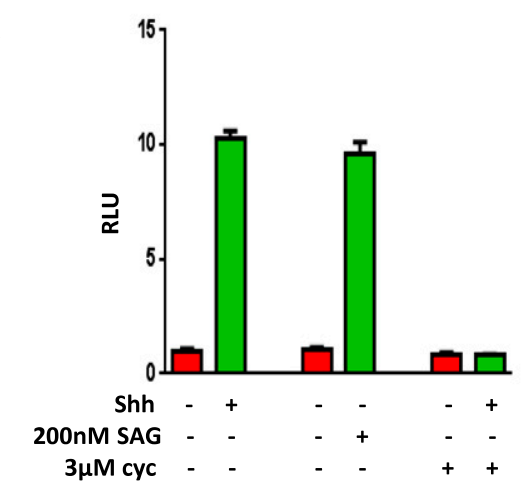

C

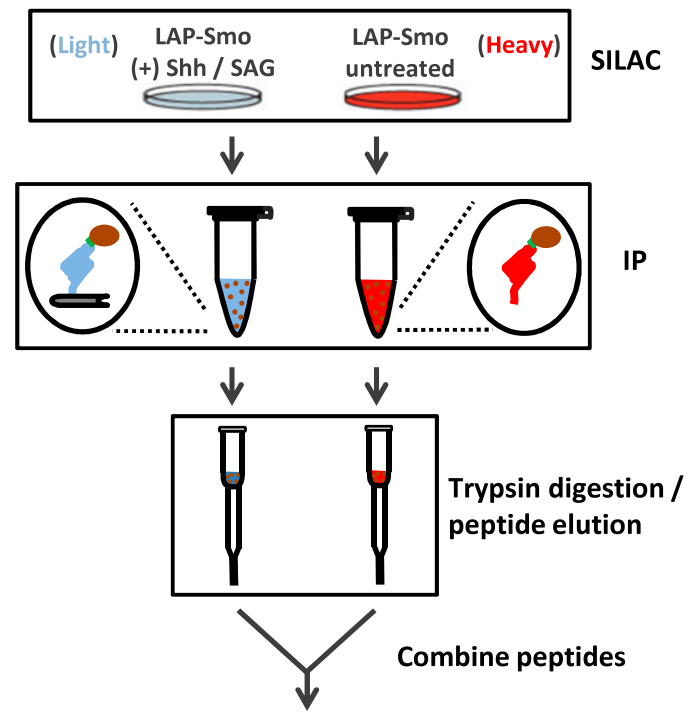

LC-MS/MS
B

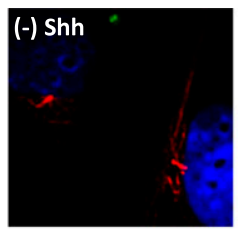

$(+)$ Shh

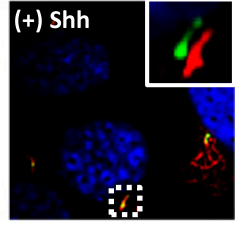

GFP (Smo) AcTub

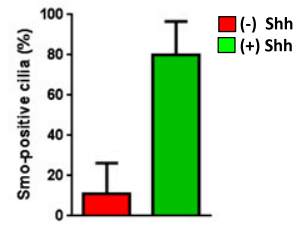

D

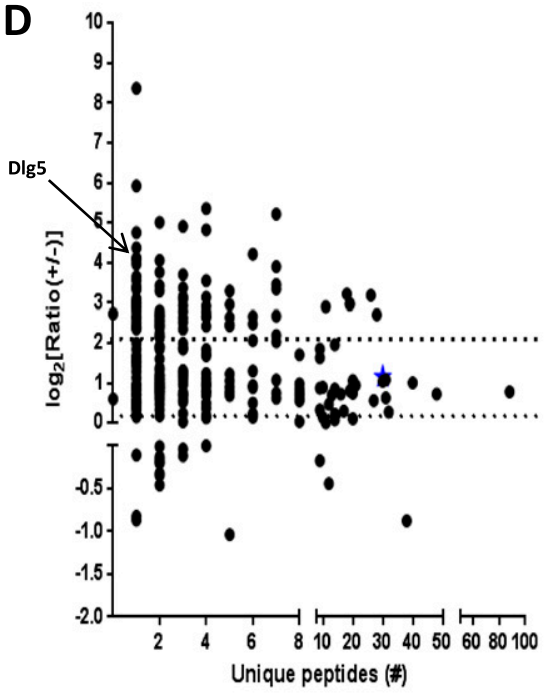

$\mathbf{F}$

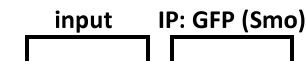

$\begin{array}{lllllllll}\text { ShhN (h) } & 0 & 1 & 7 & 19 & 0 & 1 & 7 & 19\end{array}$

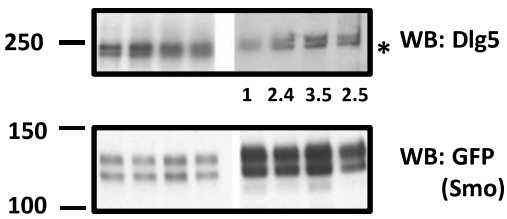

Figure 1. Activation of the Hh pathway in LAP-Smo-expressing MEFs induces the interaction of Smo and Dlg5. (A) Gli-luciferase activity in LAP-Smo MEFs treated with ShhN conditioned medium, SAG1.5, and/or cyclopamine. (B) Microscopy analysis of untreated or ShhN-stimulated LAP-Smo MEFs stained using antibodies against acetylated tubulin (red) to mark the primary cilium and GFP (green) to detect LAP-Smo. The boxed region is magnified and shown as a shifted overlay demonstrating colocalization of LAP-Smo and acetylated tubulin. The fraction of primary cilia that overlaps with Smo is quantified and plotted. All error bars are SD. $n>50$ cilia. $(C)$ A schematic representation of the SILAC-based labeling of LAP-Smo MEFs for the identification of Smo-interacting proteins by LC-MS/MS. $(D)$ A scatter plot depicting results of the quantitative comparison of proteins binding to LAP-Smo in Shh/SAG1.5stimulated and untreated MEFs. The logarithmic ratios of protein abundance are plotted against the number of unique peptides detected for each protein. The position of Smo on the graph is denoted by a star symbol in blue. ShhNp/SAG treatment significantly enriches the amount of Dlg5 binding to Smo. $(E)$ Coimmunoprecipitation with an anti-GFP matrix of lysates from HEK293T cells transfected with LAP-Smo and Dlg5-3xFlag, demonstrating specific binding of Dlg5 and Smo. (F) Coimmunoprecipitation with an antiGFP matrix of lysates from LAP-Smo2 MEFs stimulated with ShhN conditioned medium for the indicated times and incubated with 1 mM DSP cross-linker. Shh stimulation increases the amount of Dlg5 bound to Smo. $\left({ }^{\star}\right)$ Nonspecific bands.

catalytically inactive. PDZ domains are 80-90 amino acids in length and usually bind at the extreme $\mathrm{C}$ termini of target proteins (Hung and Sheng 2002), whereas SH3 domains are smaller (60 amino acids long) and bind hydrophobic PXXP motifs or related sequences (Sparks et al. 1996, 1998; Mayer 2001). GK domains are essential for function of MAGUK proteins, but little is known about the protein-protein interactions that they may mediate. Deletion of the GK domain in the Drosophila Dlg tumor suppressor inactivates the protein and results in imaginal disc tumors and reduced survival (Woods et al. 1996). To map the interaction of Dlg5 
with Smo, we coexpressed Dlg5 domains (Fig. 2A) together with Smo in HEK293T cells. The most C-terminal PDZ (PDZ4) and the GK domains were sufficient to mediate Smo binding when expressed individually or in combination with other domains (Fig. 2B), with the strongest interactions seen for GK-containing constructs.
To map the region of the Smo cytoplasmic tail (SmoCT) necessary for interaction with Dlg5, we generated 19 mutants spanning the length of the SmoCT, each containing a stretch of 10 consecutive amino acids mutated to alanine (Supplemental Fig. S3). We initially assayed the ability of these Smo mutants to bind the GK domain of Dlg5 and found that SmoCT-10A-17 exhibited the stron-
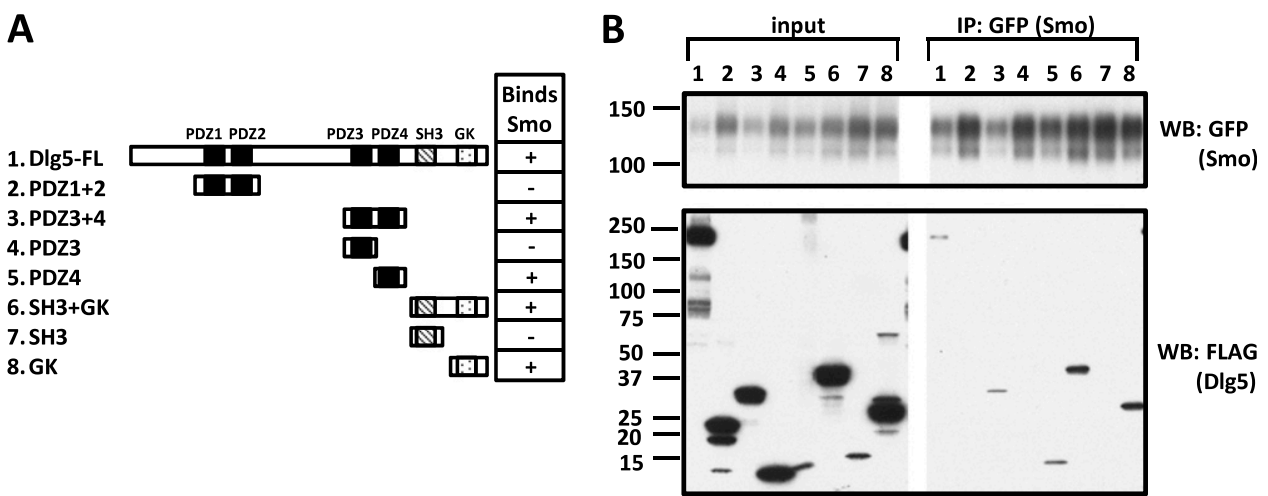

C

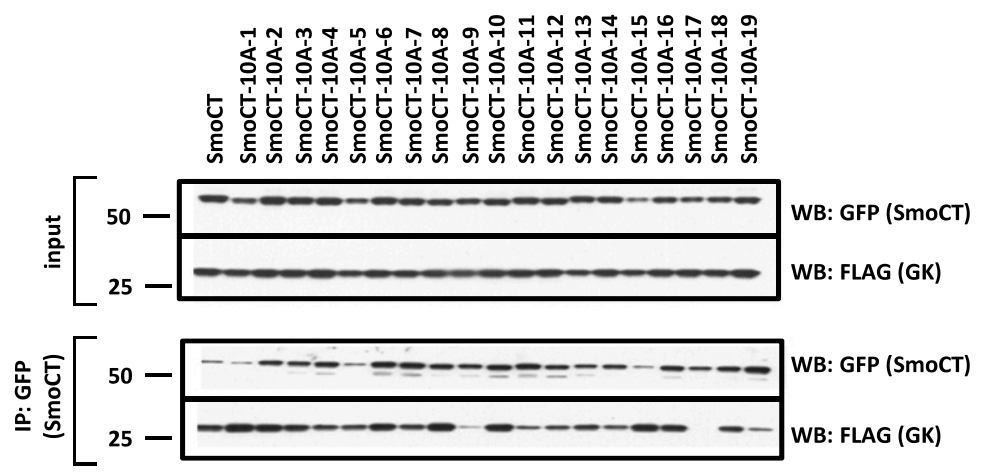

D

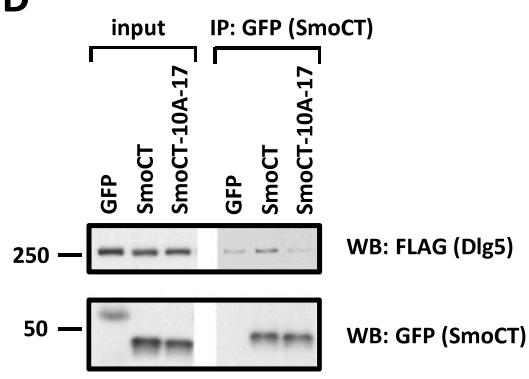

E

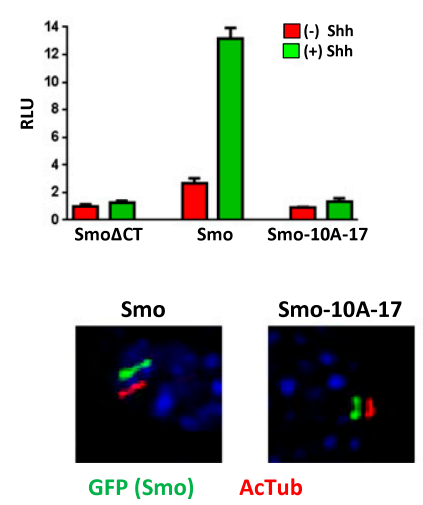

Figure 2. Mapping domains required for the interaction of Smo and Dlg5. (A) Schematic representation of Dlg5 domain organization and individual domains fragments used for mapping Smo interaction sites. (B) Lysates of HEK293T cells transfected with LAP-Smo and the indicated Dlg5 domain constructs were coimmunoprecipitated with an anti-GFP matrix. Only Dlg5 fragments containing PDZ4 or GK domains interacted with Smo. $(C)$ Coimmunoprecipitation with an anti-GFP matrix of lysates from HEK293T cells transfected with the indicated YFP-SmoCT alanine mutants and Dlg5-GK-3xFlag. Dlg5-GK bound to all SmoCT mutants except for SmoCT-10A-17. (D) Loss of specific interaction between full-length Dlg5 and SmoCT-10A-17 was assayed in HEK293T cells transfected with YFP-SmoCT/ SmoCT-10A-17 and Dlg5-3xFlag. Dlg5 bound the SmoCT but not SmoCT-10A-17. (E) Gli-luciferase activity in Smo ${ }^{-/-}$MEFs transfected with the indicated Smo constructs and stimulated with ShhN conditioned medium. Smo-10A-17 behaves functionally similarly to Smo lacking the cytotail (Smo $\Delta \mathrm{CT}$ ), being incapable of rescuing defective Hh response in $S m o^{-1-} \mathrm{MEFs}^{-}(F)$ Microscopy analysis of NIH3T3 fibroblasts transfected with the indicated Smo constructs, fixed with 4\% paraformaldehyde, and stained using antibodies against acetylated tubulin (red) and GFP (green) to visualize transfected Smo. Images shown are shifted overlays demonstrating overlap of Smo/Smo-10A-17 and acetylated tubulin. 
gest defect in binding (Fig. 2C). We confirmed that SmoCT-10A-17 also failed to bind full-length Dlg5 (Fig. 2D), suggesting that amino acid residues 585-597 are essential for any physical association of Smo with Dlg5. This Smo-10A-17 mutant that fails to bind Dlg5 is also functionally defective in transducing the Hh signal when introduced into $\mathrm{Smo}^{-1-}$ cells (Fig. 2E) yet accumulates in the primary cilium when overexpressed in NIH3T3 fibroblasts (Fig. 2F). These results suggest that Smo's ability to traffic into the primary cilium is independent of its interaction with Dlg5.

Dlg5 is a positive regulator of th signaling

As the inability of Smo to bind Dlg5 correlates with the impairment of Smo-mediated transduction of the $\mathrm{Hh}$ signal (Fig. 2D,E), we compared the state of Hh pathway activation in MEFs derived from Dlg5 knockout mice $\left(D \lg 5^{-/-} \mathrm{MEFs}\right)$ and control MEFs from $D \lg 5^{+/-}$mice. We found that expression of the Glil protein, encoded by the Hh transcriptional target Gli1, was impaired in Shhtreated $D \lg 5^{-/-}$MEFs (Fig. 3A), and this defect was rescued by reintroduction of exogenous LAP-Dlg5 into $D \lg 5^{-/-}$ MEFs (Fig. 3B). Two different small molecule Smo agonists-SAG1.5 and activating oxysterols [a combination of 20(S) and 22(S)-hydroxycholesterol]_also failed to induce Gli1 expression in Dlg5 $5^{-/-}$MEFs (Fig. 3C). Furthermore, siRNA-mediated depletion of Dlg5 in $\mathrm{Ptch}^{-/}$MEFs resulted in a 50\% reduction in Gli1 protein levels, whereas a similar depletion had a minimal effect on Glil in Sufu ${ }^{-1-}$ MEFs (Fig. 3D). Importantly, there were no significant differences in the frequency of ciliated cells in $D \lg 5^{+/-}$and $D \lg 5^{-1-}$ MEFs (Fig. 3E,F). Consistent with ciliary accumulation of the Smo-10A-17 mutant (Fig. 2E), which is defective in Dlg5 binding, the absence of endogenous Dlg5 did not impair ciliary accumulation of Smo in response to Shh stimulation (Fig. 3G,H). Taken together, these results suggest that Dlg5 binding to Smo may regulate Hh pathway activity at a level upstream of Sufu without affecting Smo ciliary trafficking.

\section{Dlg5 localizes to the basal body}

Dlg5 has been shown to associate with $\mathrm{N}$-cadherin in cytoplasmic vesicles and at adherens junctions (Nechiporuk et al. 2007). In addition, we noted significant colocalization of overexpressed LAP-Dlg5 specifically with the $\gamma$-tubulinenriched basal body at the base of the primary cilium but not with the adjacent daughter centrosome in NIH3T3 fibroblasts (Fig. 4A). To validate association of endogenous Dlg5 with the basal body, we fractionated subcellular organelles from LAP-Smo2 MEFs using sucrose density gradient centrifugation and analyzed by Western blot the fractions corresponding to $40 \%-70 \%$ sucrose, which include centrosomes but exclude less dense cytoplasmic vesicles and plasma membrane. We found that endogenous Dlg5 cosedimented with $\gamma$-tubulin in lysates from these unstimulated LAP-Smo2 MEFs (Fig. 4B,C) as well as MEFs stimulated with Shh (Fig. 4D,E). We also analyzed these sucrose fractions for Lamin-A/C (a marker for the nucleus), calnexin (a marker for the endoplasmic reticulum), and acetylated tubulin and failed to detect enrichment of these proteins in the $\gamma$-tubulin-containing fractions (data not shown). These results suggest that $\mathrm{D} \lg 5$ is a resident protein of the basal body whose localization at this organelle is independent of the state of activation of the Hh pathway. In addition to Dlg5, LAP-Smo also partially cofractionates with $\gamma$-tubulin, raising the intriguing possibility that $\mathrm{Hh}$ signaling may be influenced by an interaction between Smo and Dlg5 at the basal body prior to Smo entry into the primary cilia.

\section{Kif7 is a basal body-associated protein that binds both Smo and Dlg5}

The kinesin-like protein Kif7 is another mammalian $\mathrm{Hh}$ pathway component that has been reported to localize to the base of the cilium (Endoh-Yamagami et al. 2009; Liem et al. 2009), with Hh-induced accumulation at the ciliary tip. We confirmed the localization of Kif7 at the ciliary base and further demonstrated its colocalization with $\gamma$-tubulin at centrosomes (Fig. 5A) in $D \lg 5^{+/-}$cells, which are essentially normal in their response to Shh stimulation. In addition, we observed a twofold increase in accumulation of Kif7 at the ciliary tip upon Shh stimulation (Fig. 5B,C). Given the colocalization of Kif7 and Dlg5 at the basal body, we tested their physical interaction by coexpressing LAP-Dlg5 and Kif7-3xFlag in HEK293T cells and were able to demonstrate that Dlg5 specifically coimmunoprecipitates Kif7 (Fig. 5D) and that Kif7 specifically coimmunoprecipitates Dlg5 (Fig. 5E). We further found that, like Dlg5, Kif7 interacts with the SmoCT (Fig. 5F), consistent with previous studies demonstrating an interaction between Smo and Kif7 (EndohYamagami et al. 2009; Yang et al. 2012).

Kif7 is a member of the kinesin superfamily of proteins, most of which comprise four distinct structural domains: an N-terminal motor domain with the ability to bind nucleotides and microtubules, a short neck that serves as a linker, a stalk region that contains multiple coiled-coil domains, and a globular tail that generally functions as the cargo-binding domain (depicted in Supplemental Fig. S4A; Katoh and Katoh 2004; Klejnot and Kozielski 2012). By coexpression of these distinct domains in HEK293T cells, we were able to map the neck domain of Kif7 as the site of interaction with Dlg5 (Supplemental Fig. S4B) and distinct sites in the neck and stalk domains of Kif7 as the sites of interaction for the SmoCT (Supplemental Fig. S4C).

To map the corresponding determinants in the SmoCT that are required for Smo to interact with Kif7, we coexpressed in HEK293T cells the previously described SmoCT alanine block mutants and Kif7. We found that, in contrast to Dlg5, the interaction with Kif7 is disrupted by multiple SmoCT-10A mutants (Supplemental Fig. S5A), suggesting that multiple nonlinear determinants of the SmoCT contribute to interaction with Kif7. Interestingly, despite poor conservation of the cytoplasmic tails of Drosophila and vertebrate Smo, SmoCT residues 644-653, which when mutated in SmoCT-10A-12 abolish interaction with Kif7, are part of the region that most closely aligns to the region in Drosophila Smo that is 
A

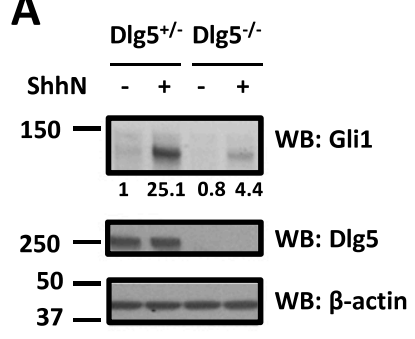

D

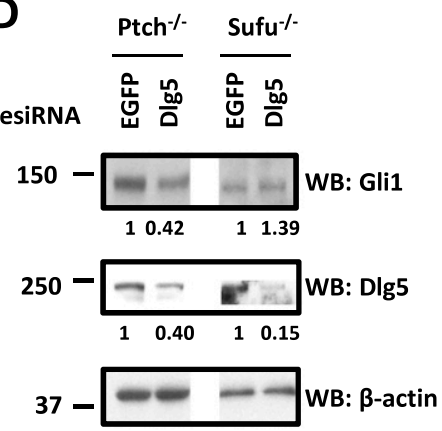

G

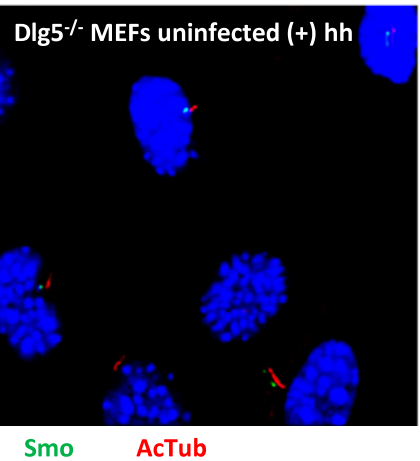

B

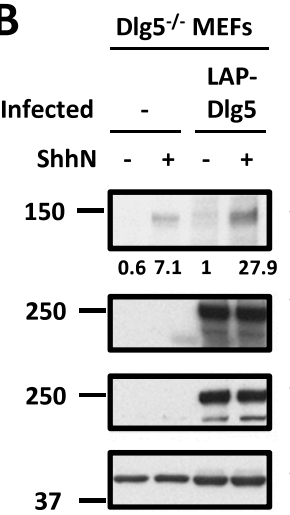

E

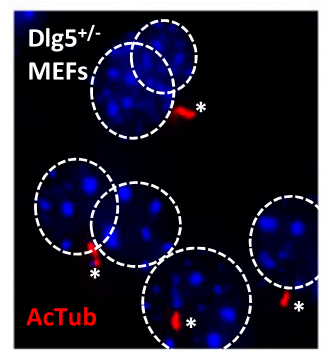

WB: $\beta$-actin
C
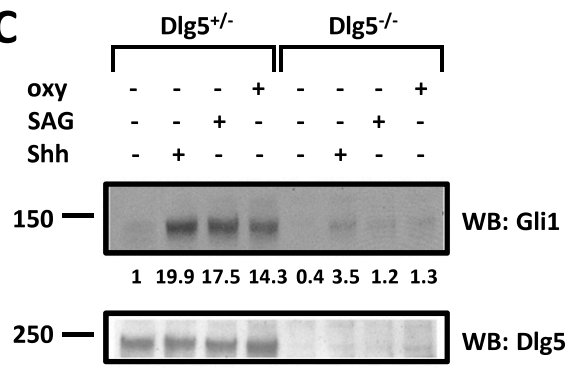

$30-2$ WB: $\beta$-actin
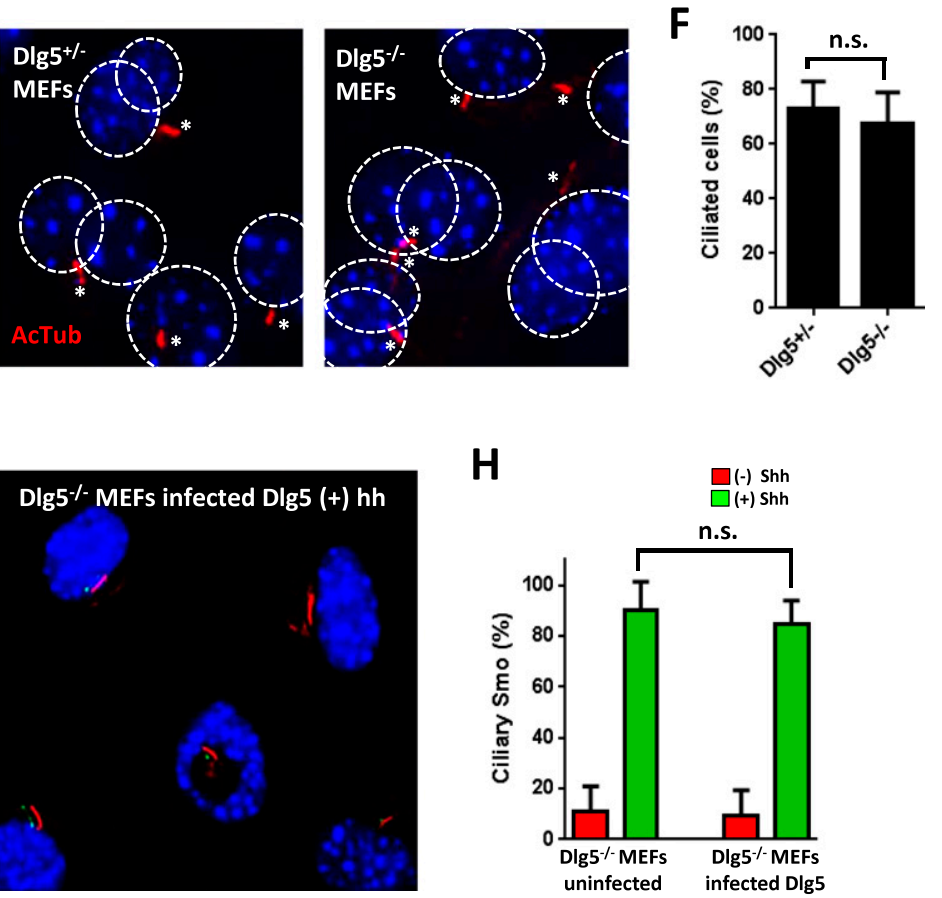

Figure 3. Dlg5 loss compromises Hh signal response without affecting the formation of the primary cilium or the ciliary accumulation of Smo. (A) Western blot (WB) analysis of Gli1 levels in Shh-stimulated $D l g 5^{+/-}$and $D l g 5^{-1-}$ MEFs. Shh induces the accumulation of Gli1 in $D l g 5^{+/-}$cells, whereas Dlg5 $5^{-1-}$ cells exhibit significantly reduced Glil protein levels in response to Shh. (B) Western blot analysis of Gli1 levels in Shh-stimulated Dlg5 ${ }^{-1-}$ MEFs either uninfected or infected with LAP-Dlg5. Introduction of Dlg5 into Dlg5 $5^{-/-}$cells via retroviral transduction restores Glil protein accumulation in cells stimulated with Shh. $(C) D l g 5^{+/-}$and $D l g 5^{-/-}$MEFs were stimulated for $24 \mathrm{~h}$ with the indicated treatments, and Glil levels were analyzed by Western blot. Neither ShhN conditioned medium, 200 nM SAG1.5, nor a combination of $5 \mu \mathrm{M} 20(\mathrm{~S}) / 22(\mathrm{~S})$-hydroxycholesterol was capable of inducing Gli1 in $D \lg 5^{-/-}$MEFs to levels comparable in $D l g 5^{+/-} \mathrm{MEFs}$. $(D)$ Western blot analysis of the effect of Dlg5 knockdown on Glil levels in unstimulated Ptch ${ }^{-/-}$and Sufu ${ }^{-/-}$MEFs. Depletion of Dlg5 in Ptch $^{-1-}$ MEFs resulted in a decrease in Glil protein, but a similar knockdown of Dlg5 in Sufu ${ }^{-1-}$ MEFs had no appreciable effect on Glil. $(E, F)$ Microscopy analysis of $D \lg 5^{+/-}$and $D \lg 5^{-/-}$MEFs that were subjected to overnight serum starvation and subsequently stained with an antibody against acetylated tubulin (red) to label primary cilia. DAPI-labeled nuclei are outlined using white dashed circles, and primary cilia are indicated by asterisks. The fraction of nuclei associated with a primary cilium in each cell line is quantified in $F$. All error bars are SD. $n>$ 95 nuclei. Data were analyzed using a two-tailed unpaired $t$-test $(P=0.46)$. There was no significant difference in the frequency of ciliation in $D \lg 5^{+-}$and Dlg5 $5^{-1-}$ cells. $(G, H)$ Microscopy analysis of Shh-stimulated uninfected or LAP-Dlg5-infected Dlg5 ${ }^{-/-}$MEFs stained with antibodies against acetylated tubulin (red) and Smo (green). Images shown are shifted overlays. The fraction of primary cilia that overlaps with Smo is quantified and plotted in $H$. All error bars are SD. $n>43$ cilia. Data were analyzed using a two-tailed unpaired $t$-test $(P=0.36)$. The fraction of cilia that contained Smo in response to Shh was not significantly different in the two cell populations.

required for interaction with Cos2 (Supplemental Fig. S5B; Lum et al. 2003). It is also noteworthy that SmoCT10A-17, which fails to bind Dlg5, retains the ability to interact with Kif7, suggesting that Dlg5 and Kif7 in- teraction with Smo does not need to be mutually exclusive. Finally, we also determined that the PDZ4 and GK domains of Dlg5 are sufficient for binding Kif7 (Supplemental Fig. S5C). 
Chong et al.

A
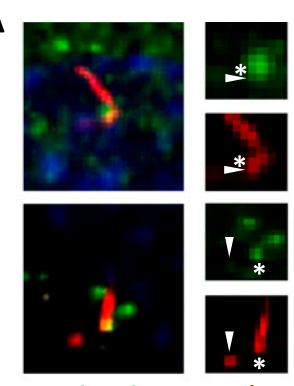

GFP (Dlg5) AcTub / yTub

C

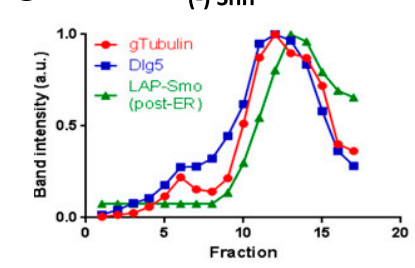

E

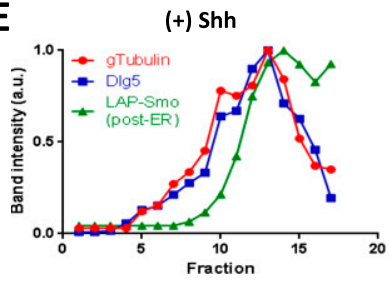

B

LAP-SMO2 MEF (-) Shh

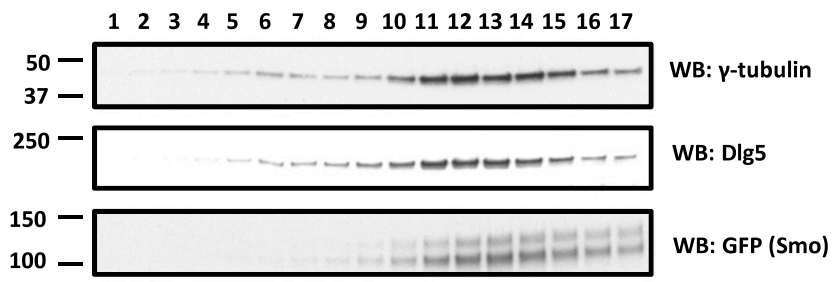

D

LAP-Smo2 MEF (+) Shh

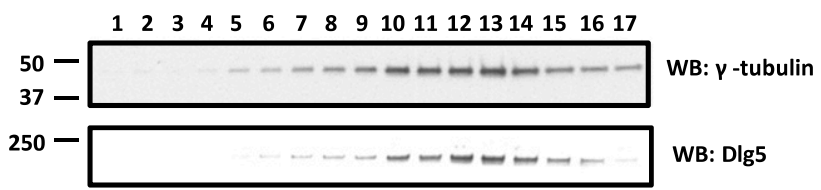

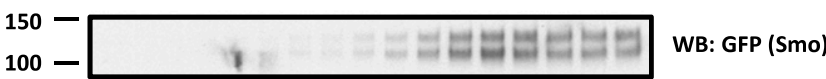

Figure 4. Dlg5 localizes to the basal body. (A) Microscopy analysis of NIH3T3 cells transfected with LAP-Dlg5 and stained using antibodies against acetylated tubulin and $\gamma$-tubulin (both red) and GFP (green) to label LAP-Dlg5. Dlg5 colocalized with the basal body immediately proximal to the cilium (asterisk) but not with the daughter centrosome (arrowhead). (B-E) Western blot (WB) analysis of the distribution of Dlg5 and GFP (LAP-Smo) in centrosome-enriched sucrose fractions of LAP-Smo2 MEFs either untreated (B) or stimulated with Shh $(D)$. Intensities of Western blot bands were quantified using ImageJ software (National Institutes of Health) and are plotted in $C$ and $E$. In both unstimulated and Shh-treated cells, the peaks of $\gamma$-tubulin overlapped with those of Dlg5 and post-ER Smo.

Smo and Dlg5 are necessary for Shh-induced accumulation of Kif7 at the ciliary tips

Kif7 accumulation at the ciliary tip is correlated with $\mathrm{Hh}$ pathway activation, and a Kif7 mutant that fails to accumulate at this site causes defective Hh signal transduction (Liem et al. 2009). As we do not see Dlg5 accumulation at the ciliary tip, it is likely that any functionally meaningful interaction between Dlg5 and Kif7 would occur prior to Kif7 entry into the cilium. Accordingly, we tested the effect of Dlg5 function on Hhinduced accumulation of Kif7 at the ciliary tip and found that in $D \lg 5^{-1-}$ cells, which lack all Dlg5 function, the basal level of Kif7 localized to the ciliary tip did not increase significantly upon Shh stimulation (Fig. 6A-C); this defect was rescued by retroviral transduction of $D \lg 5^{-1-}$ cells with a Dlg5 expression construct (Fig. 6DF) but not by a similar construct lacking the domains necessary for binding both Smo and Kif7 (Dlg5- $\triangle \mathrm{PDZ} 4, \Delta \mathrm{GK}$ ) (Supplemental Fig. S6). To further investigate the dependence of Kif7 ciliary accumulation on Smo, we examined Kif7 localization in $\mathrm{Smo}^{-1-}$ and LAP-Smo2 MEFs. We noted no increase in the amount of Kif7 at the ciliary tip in Shh-treated $\mathrm{Smo}^{-1-}$ MEFs (Fig. 6G-I) but did observe a rescue of Hh-induced accumulation of Kif7 at the ciliary tip when these cells were stably transduced with a retroviral construct for expression of Smo (LAPSmo2 MEFs) (Fig. 6J-L).

\section{Dlg5 regulates Gli2 ciliary accumulation but not Gli3 processing}

Gli2 and Gli3, respectively, are the major transcriptional activating and repressing transcriptional effectors of $\mathrm{Hh}$ signaling. Full-length Gli3 (Gli3FL) is processed into a truncated repressor (Gli3R) in the absence of Hh (Wang et al. 2000); Hh stimulation not only blocks proteolytic cleavage of Gli3 but also induces the accumulation of Gli2 at the tip of the primary cilium /Chen et al. 2009; Kim et al. 2009), an event that precedes Gli2-mediated transcriptional activation in the nucleus. Although Smo is required for reducing formation of Gli3R and for activating Gli2, both of which increase Hh-induced transcription of pathway targets, Kif7 has been implicated in both pathway suppression via formation of Gli3R (Cheung et al. 2009; Endoh-Yamagami et al. 2009) and pathway activation (Cheung et al. 2009; Liem et al. 2009), presumably via Gli2. The ratio of Gli3FL/Gli3R as well as the ciliary accumulation of Gli2 thus serve as functional readouts of Hh pathway activity and can be analyzed to determine how the defects in $D l g 5^{-1-}$ cells impact the pathway. We observed some Gli2 localization at the ciliary tip in 
A

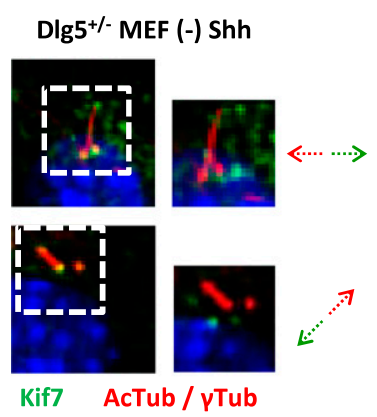

D

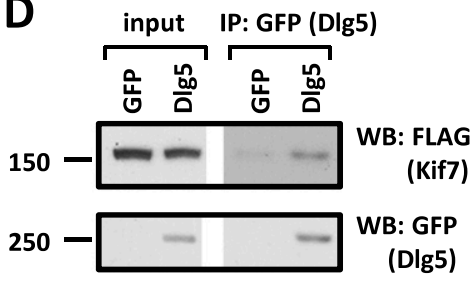

B

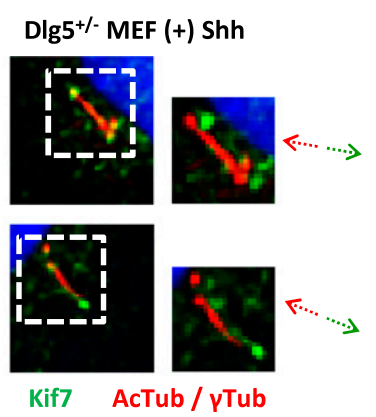

$E$

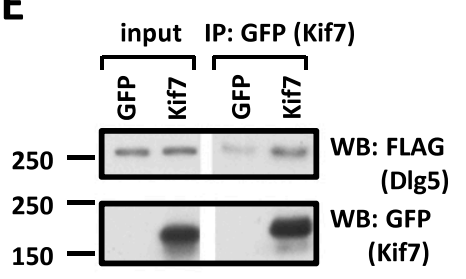

C

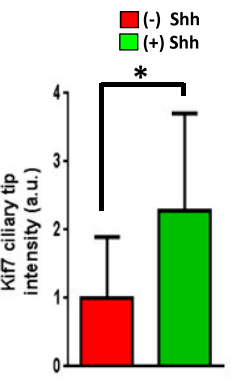

$\mathbf{F}$

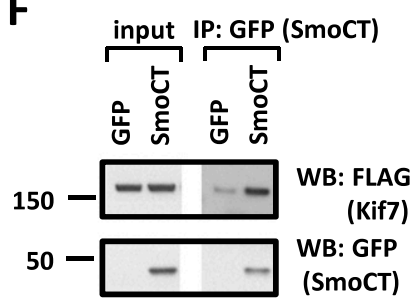

Figure 5. Kif7 is a basal body-localized protein that interacts with both Smo and Dlg5. (A-C) Microscopy analysis of $D l g 5^{+/-}$MEFs untreated $(A)$ or stimulated with Shh $(B)$ and stained using antibodies against acetylated tubulin and $\gamma$-tubulin (both red) and Kif7 (green). The pixel intensity of Kif7 at the tip of each cilium was quantified using ImageJ, and the mean Kif7 intensities for each treatment are plotted in $C$. All error bars are SD. $n>20$ cilia. Data were analyzed using a two-tailed unpaired $t$-test $\left(\left[{ }^{\star}\right] P=0.0003\right)$. Kif7 constitutively localized to the basal body and also accumulated at the ciliary tip in response to Shh. $(D-F)$ Coimmunoprecipitation with an anti-GFP matrix of lysates from HEK293T cells transfected with either LAP-Dlg5 and Kif7-3×Flag $(D)$, Kif7-EGFP and Dlg5-3xFlag $(E)$, or YFP-SmoCT and Kif7-3xFlag $(F)$. Dlg5 specifically coimmunoprecipitated Kif7 and vice versa; Kif7 was also found to specifically bind the SmoCT.

unstimulated $D \lg 5^{-1-}$ MEFs, as also reported in wild-type MEFs (He et al. 2014), but noted that Hh stimulation failed to induce any significant increase in ciliary Gli2. Importantly, a Hh-dependent increase in accumulation of Gli2 at the ciliary tip was restored by introduction of LAP-Dlg 5 into these cells (Fig. 7A,B). In addition, we failed to observe any increase in Gli2 accumulation in the nucleus in Hhstimulated Dlg5 $5^{-1-}$ MEFs (Fig. 7C). Conversely, we noted that Hh stimulation blocked the proteolytic processing of Gli3FL into its repressor form in both the absence and presence of Dlg5, resulting in a fourfold increase in Gli3FL/ Gli3R ratios in both Hh-treated $D \lg 5^{-1-}$ MEFs and cells expressing exogenous LAP-Dlg5 (Fig. 7D,E).

As ciliary cAMP generated by Gpr161 (Mukhopadhyay et al. 2013) may activate PKA that is required for Gli processing to form a repressor, we examined the effect of Dlg5 function on Hh-stimulated ciliary exit of Gpr161. Strikingly, we found in Dlg5 $5^{-1-}$ MEFs that Hh stimulation remained fully effective in causing Gpr161 removal from the cilium (Fig. 7F,G), suggesting that the inactivation of PKA does not require Dlg5. Taking into consideration the prominent localization of PKA at the base of the cilium (Tuson et al. 2011), we investigated whether $\mathrm{CK} 1 \alpha$, another kinase implicated in Gli repressor formation (Pan et al. 2006), also localizes to the basal body. Intriguingly, we observed colocalization of endogenous $\mathrm{CK} 1 \alpha$ and $\gamma$-tubulin in both untreated and Hh-treated $D \lg 5^{+/-}$MEFs (Fig. $7 \mathrm{H}$ ), suggesting constitutive CK $1 \alpha$ localization at the basal body independent of the state of $\mathrm{Hh}$ pathway activation. Consistent with the lack of Dlg5 involvement in the Hh-induced exit of Gpr161 from the cilium and the suppression of Gli3R formation, we found that $\mathrm{CK} 1 \alpha$ also localizes at the basal body in the absence of Dlg5 (Supplemental Fig. S7). These data suggest that, despite overall impairment of $\mathrm{Hh}$ response in a $\mathrm{Dlg} 5^{-/-}$ setting, the activities of components involved in regulating Gli3R formation remain fully intact.

\section{Discussion}

Hh-induced changes in gene transcription result from suppression of the proteolytic processing that generates Gli repressor (predominantly Gli3) (Wang et al. 2000) and from activation and nuclear accumulation of full-length Gli proteins (predominantly Gli2) (Chen et al. 2009; Kim et al. 2009). These effects of Hh pathway activation on Gli repressor formation and Gli activation both require Smo. In contrast, we found that although the Smo-interacting protein Dlg5 is required for Gli activation, it is not required for Hh-induced suppression of Gli repressor formation. Our observations thus suggest that Smo activity bifurcates into a Dlg5-independent arm, acting via suppression of Gli repressor formation, and a Dlg5dependent arm, which is associated with accumulation of Kif7 and Gli at the ciliary tip and Gli accumulation in the nucleus. Interestingly, Kif7 appears to have opposing roles in these two arms of Hh response. Gli3R processing is lost in Kif7 mutants (Cheung et al. 2009; Endoh-Yamagami et al. 2009), implying a role in promoting repressor formation, but Kif7 loss also reduces pathway activation, suggesting a positive role for Kif7 function in the Gli activation arm of $\mathrm{Hh}$ response. This dual role of Kif7 in Hh response parallels the dual role of Cos2 in Drosophila Hh response (Sisson et al. 1997; Wang and Holmgren 
Chong et al.

A

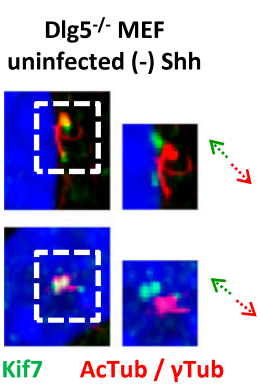

D
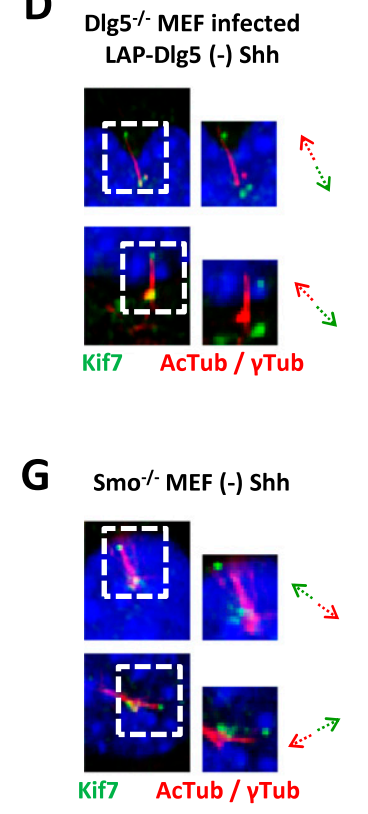

\section{J}

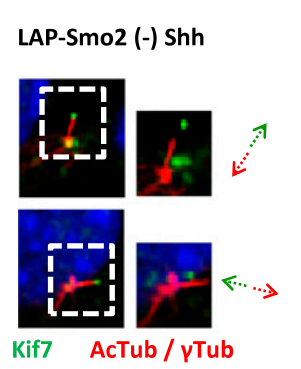

B

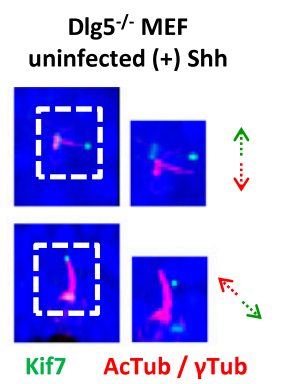

E

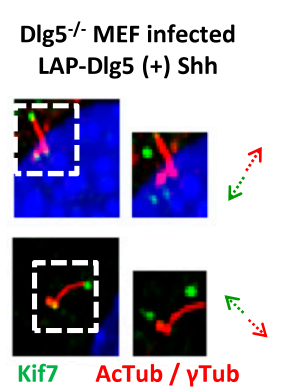

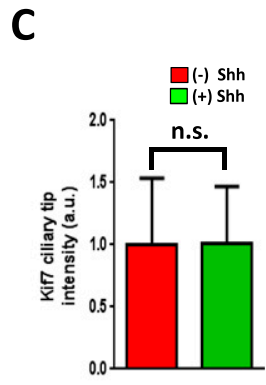
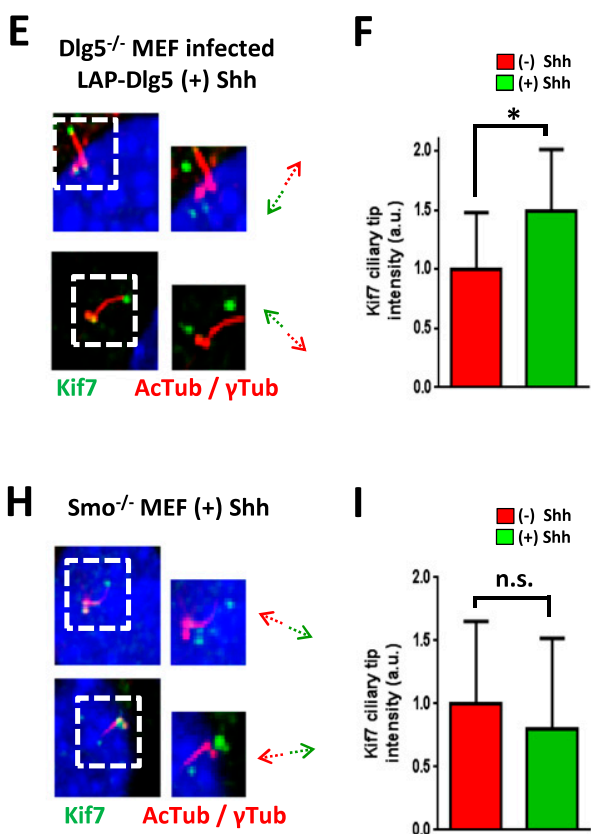

K

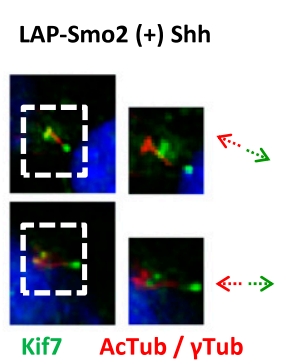

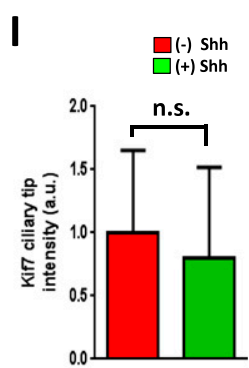

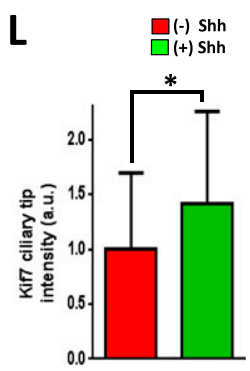

Figure 6. Shh-induced accumulation of Kif7 at the tip of the primary cilium requires both Smo and Dlg5. (A$C)$ Microscopy analysis of $D \lg 5^{-1-}$ MEFs untreated $(A)$ or stimulated with Shh $(B)$. Mean pixel intensities of Kif7 (green) at the ciliary tips were quantified and are plotted in $C$. $n>46 ; P=0.95$. $(D-F)$ Microscopy analysis of LAP-Dlg5-infected Dlg5 ${ }^{-1-}$ MEFs untreated $(D)$ or stimulated with Shh $(E)$. Mean pixel intensities of Kif7 (green) at the ciliary tips were quantified and are plotted in $F . n>60 ;\left(^{\star}\right) P=3 \mathrm{E}-07 .(G-I)$ Microscopy analysis of $S \mathrm{mo}^{-1-}$ MEFs untreated $(G)$ or stimulated with Shh $(H)$. Mean pixel intensities of Kif7 (green) at the ciliary tips were quantified and are plotted in $I . n>8 ; P=0.72$. $(J-L)$ Microscopy analysis of $L A P$-Smo2 MEFs untreated $(J)$ or stimulated with Shh $(K)$. Mean pixel intensities of Kif7 (green) at the ciliary tips were quantified and are plotted in $L . n>27 ;\left(^{\star}\right) P=0.022$. All samples were stained using antibodies against acetylated tubulin and $\gamma$-tubulin (both red) and Kif7 (green). Shifted overlays of all boxed regions are shown, and the direction of displacement of the green channel with respect to the red channel for individual shifted overlays is indicated by the arrows. All error bars are SD, and data were analyzed using a two-tailed unpaired $t$-test. Shh failed to induce Kif7 accumulation at the ciliary tips in $D \lg 5^{-/-}$ MEFs, but introduction of LAP-Dlg5 into these cells restored Shh-induced Kif7 ciliary accumulation. Similarly, Kif7 accumulation at the ciliary tips was impaired in Shh-treated $\mathrm{Smo}^{-1-} \mathrm{MEFs}$, but LAP-Smo2 MEFs exhibited normal Kif7 accumulation in the cilium in response to Shh.
2000; Wang et al. 2000; Lum et al. 2003). In these studies, Cos2 loss was found to induce constitutive Hh pathway activity but also limit the level of this activity, thus revealing both positive and negative roles in pathway regulation.

Within the Dlg5-independent arm, as revealed in cells lacking Dlg5 function, we observed Hh-induced Smo ciliary accumulation and ciliary exit of Gpr161 (Fig. 8). Gpr161 signaling via $\mathrm{G} \alpha_{\mathrm{s}}$ and adenylyl cyclase increases cAMP in the cilium (Mukhopadhyay et al. 2013). Given the free passage of small molecules between the cilium and cytoplasm (Breslow et al. 2013; Lin et al. 2013), ciliary cAMP might be expected to activate the fraction of PKA that is prominently localized at the base of the cilium
(Liem et al. 2012; Mukhopadhyay et al. 2013). The additional presence of $\mathrm{CK} 1 \alpha$ at the basal body, as noted above (Fig. 7H; Supplemental Fig. S7), suggests that the ciliary base may be the site of PKA-mediated phosphorylation events that in turn prime $\mathrm{CK} 1 \alpha$-mediated phosphorylation events and that together contribute to Gli repressor formation (Wang et al. 2000). In this context, we note that the importance of the cilium for Gli repressor formation may be primarily as a local source of cAMP for activation of PKA at the basal body. Hh-stimulated exit of Gpr161 from the cilium would then remove the source of cAMP and reduce PKA activation, and ciliary exit of Gpr161 thus could be the critical event in Hh-mediated suppression of Gli repressor formation. Consistent with 
A

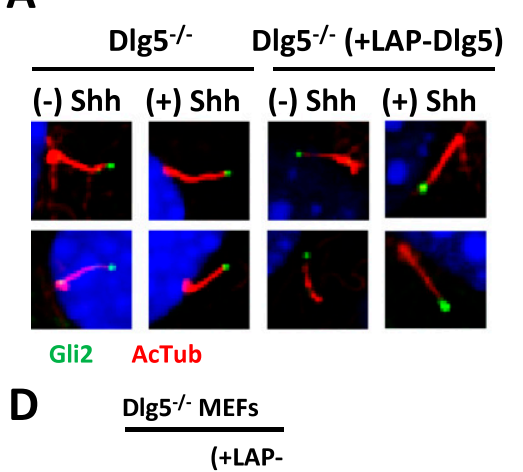

37

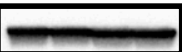

WB: $\beta$-actin

F
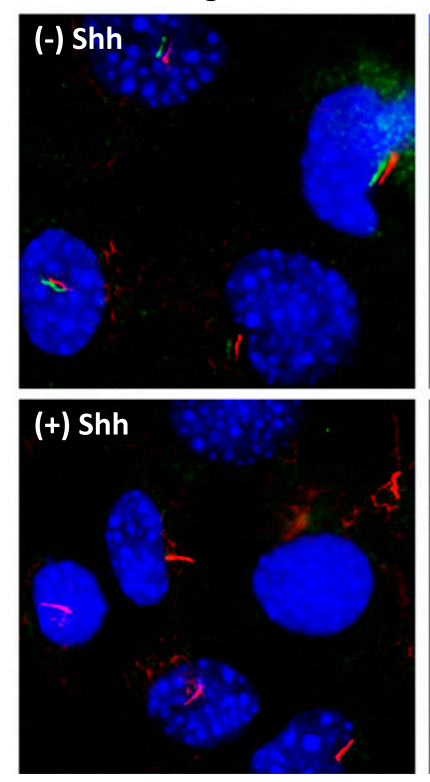

Gpr161 AcTub
B

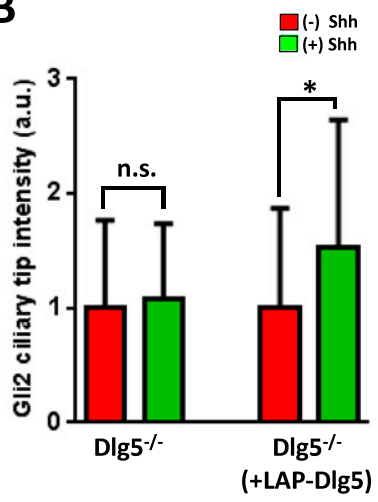

E

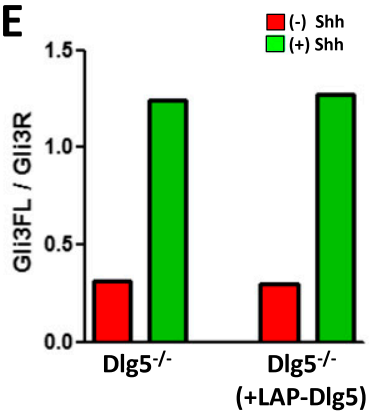

C

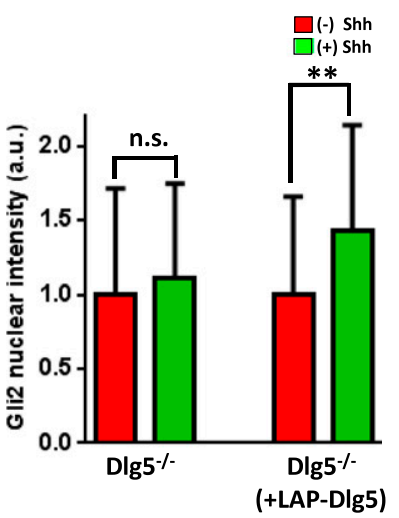

$\mathbf{G}$

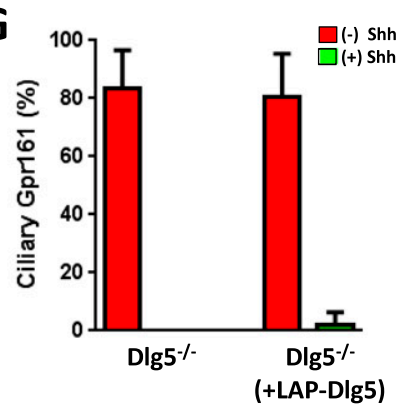

H
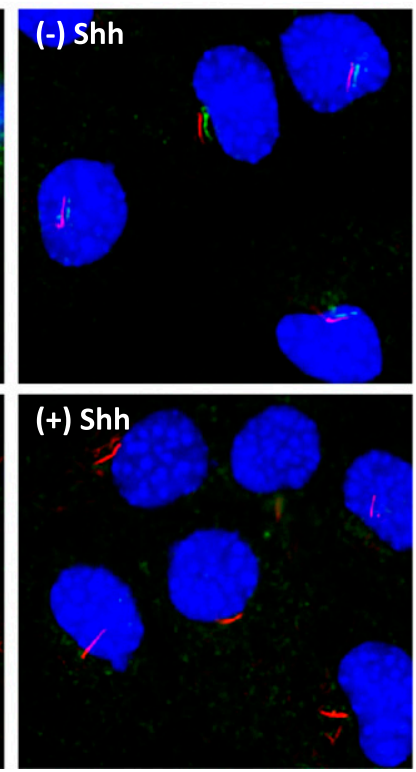
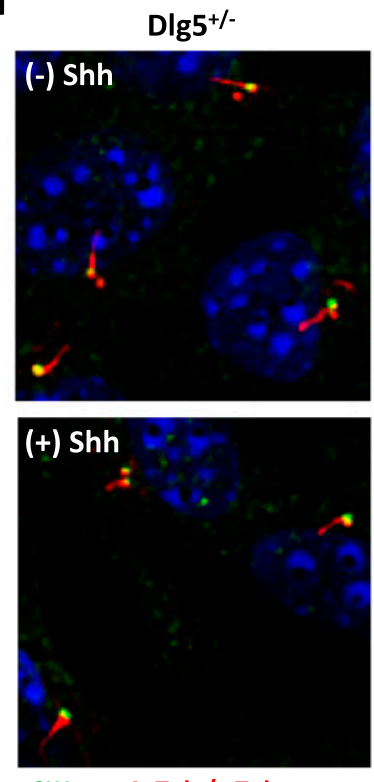

CK1 $\alpha$ AcTub / $ү$ Tub

Figure 7. Dlg5 regulates Gli2 ciliary accumulation but not Gli3 processing. $(A-C)$ Microscopy analysis of $D 1 g 5^{-1-}$ MEFs and cells transduced with LAP-Dlg5 and stained using antibodies against acetylated tubulin (red) and Gli2 (green). The pixel intensities of Gli2 at the ciliary tip and nucleus were quantified using ImageJ, and the mean Gli2 intensities for each treatment are plotted. All error bars are SD. $n>65$. (n.s.) $P=0.51 ;\left(^{\star}\right) P=0.0013$ ) for ciliary Gli2. (n.s.) $\left.P=0.57 ;{ }^{\star \star}\right) P=0.013$ for nuclear Gli2. Gli2 failed to accumulate in the cilium and nucleus of $D \lg 5^{-1-}$ MEFs in response to Shh; however, introduction of LAP-Dlg5 into these cells restored Shh-induced accumulation of Gli2 in the cilium and nucleus. $(D, E)$ Western blot $(\mathrm{WB})$ analysis of Gli3FL and Gli3R levels in $D \lg 5^{-1-}$ MEFs and cells transduced with LAP-Dlg5. Band intensities were quantified using Image Lab software (Bio-Rad), and the ratio of Gli3FL/Gli3R intensities are plotted in $E$. The absence or presence of Dlg5 did not affect the ability of ShhNp to induce the inhibition of Gli3R formation. $(F, G)$ Microscopy analysis of $D \lg 5^{-/-}$MEFs and cells transduced with LAP-Dlg5 and stained using antibodies against acetylated tubulin (red) and Gpr161 (green). Images of cells not treated with Hh are shown as shifted overlays. The fraction of Gpr161-positive cilia is shown in G. Shh induced the ciliary exit of Gpr161 in both Dlg5 $5^{-/-}$MEFs and cells expressing exogenous Dlg5. (H) Microscopy analysis of Dlg5 $5^{+/-}$MEFs stained using antibodies against acetylated tubulin and $\gamma$-tubulin (both red) and CK $1 \alpha$ (green). One-hundred percent of basal bodies had associated CK1 $\alpha$ (30 out of 30 for Dlg5 $5^{+/-}[-] \mathrm{Hh}_{;} 33$ out of 33 for $\left.D \lg 5^{+/-}[+] \mathrm{Hh}\right)$. 
(-) Hh

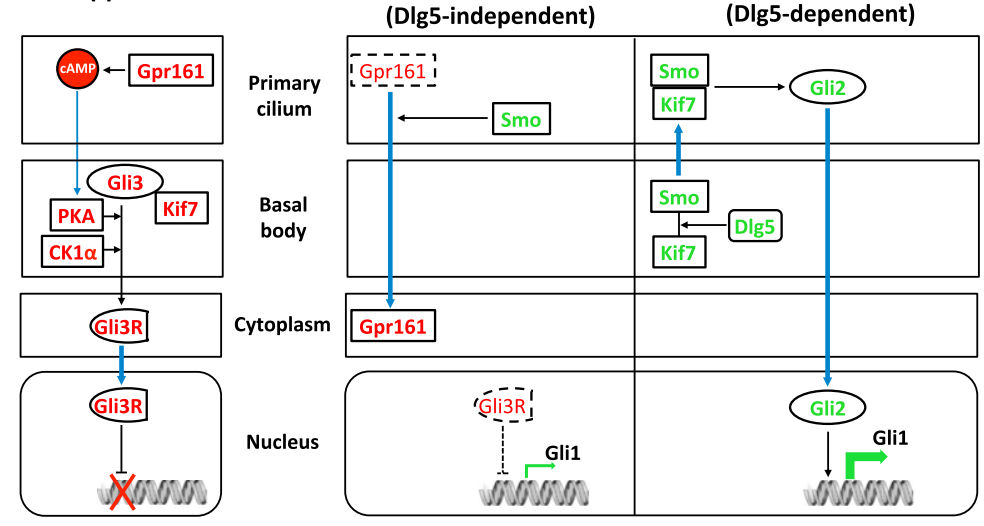

Figure 8. Model for a Dlg5-mediated bifurcation in Smo activity during $\mathrm{Hh}$ response. In the absence of $\mathrm{Hh}$, Gpr161 establishes a level of cAMP in the cilium that promotes the Kif7 and PKA/CK1 $\alpha$-dependent processing of Gli3 into Gli3R at the basal body. Upon $\mathrm{Hh}$ stimulation, in the "Dlg5-independent" arm of the pathway, Smo activation in the cilium drives the ciliary exit of Gpr161, and the consequent decrease of ciliary cAMP leads to suppression of Gli3R formation, thus alleviating transcriptional repression of Gli1 and other transcriptional targets. In the "Dlg5-dependent" arm of the pathway, Dlg5 interaction with Smo at the basal body contributes to the formation of a complex that results in accumulation of Gli2 and Kif7 in the cilium and to the activation of Gli2 and subsequent high-level $\mathrm{Hh}$ response. this model, in cells lacking Dlg5 function, we observed that Hh-induced exit of Gpr161 from the cilium and suppression of Gli repressor formation are both preserved.

In contrast, loss of Dlg5 function is associated with reduced accumulation of Gli2 in the nucleus and a block of other events associated with Gli activation, such as increased accumulation of Gli2 and Kif7 at the ciliary tip (Fig. 8). The Dlg5 protein thus appears dedicated to the Gli-activating arm of $\mathrm{Hh}$ stimulation. As Smo activation and Gli2 activation both require ciliary trafficking, it is notable that Dlg5 is prominently localized to the basal body and thus would appear to exert its effect outside of the cilium proper. In light of the role of coiled-coil domains in the targeting of proteins to the centrosome (Cohen and Parry 1990; Jakobsen et al. 2011), it is likely that the N-terminal coiled-coil domain of Dlg5 is responsible for its basal body localization. The additional interaction of Dlg5 with Kif7, which also is prominently localized to the basal body, suggests the possibility that the Dlg5 effect on Smo activity may involve formation or alteration of a Smo complex with Kif7 that then becomes competent to activate Gli2 upon entry into an activated cilium. A recent observation that Kif7 is required for the specific compartmentalization of Gli2 at the tip of the cilium (He et al. 2014) indicates that transduction of the Hh signal through the Smo/Kif7 complex might be achieved via the creation of a Kif7-mediated permissive microenvironment at the ciliary tip that allows for the subsequent accumulation of Gli2 at the distal end of the cilium. Dlg5 thus may represent a proximal factor that functions in actuation of the positive arm of Kif7 action in response to Smo activation.

The dispensability of Dlg5 for Hh-induced suppression of Gli repressor formation indicates that even $\mathrm{Dlg} 5^{-/-}$ cells undergo a significant shift in the ratio of Gli activator to Gli repressor $\left(\mathrm{Gli}^{\mathrm{A}} / \mathrm{Gli}^{\mathrm{R}}\right)$. This balance between repressor and activator is a key determinant in the transcriptional activity of $\mathrm{Hh}$ target genes. Such an alteration to the balance of $\mathrm{Gli}^{\mathrm{A}} / \mathrm{Gli}^{\mathrm{R}}$ may suffice to mediate the transcription of certain target genes despite the absence of positive Gli2 activity in a $D \lg 5^{-1-}$ setting. As an example of such an effect, the Drosophila Hh transcriptional target decapentaplegic $(d p p)$ is transcriptionally activated upon loss of the transcriptional repressor derived from $\mathrm{Ci}$, the Drosophila Gli ortholog, even in the complete absence of activated Ci (Dominguez et al. 1996; Methot and Basler 1999). The relative importance of Gli activation or Gli repressor suppression may vary among target genes and even for the same target gene in different tissues. The sustained induction of Glil expression in $D \lg 5^{-1-}$ MEFs, albeit at a reduced level, may indicate either that suppression of repressor formation suffices for low-level induction of Gli1 expression or, alternatively, that other mechanisms of Gli activation may exist that are not affected by Dlg5 loss. Sustained low-level Gli1 induction in association with intact regulation of Gli repressor formation is reminiscent of the recently reported phenotype of cells lacking function of the Evc2/Efcab7/Iqce complex (Pusapati et al. 2014), suggesting that this tripartite complex may also function primarily in the Dlg5-dependent arm of the Hh pathway to activate Gli proteins. Unlike Dlg5, however, the Evc2 complex localizes to a region at the proximal end of the cilium, where its interaction with Smo serves to spatially restrict Smo within this ciliary microcompartment.

Given the proposed role of Dlg5 in Gli activation, it may seem surprising that only $50 \%$ of $\mathrm{Dlg} 5^{-/-}$mice die perinatally (Nechiporuk et al. 2007). Although it is possible that the other four members of the Dlg protein family could contribute $\mathrm{Hh}$ signaling function in the absence of Dlg5, the N-terminal coiled-coil domain of Dlg5 is replaced in the other family members by a smaller PEST domain (Woods and Bryant 1991). Furthermore, the PDZ4 domain in Dlg5 that interacts with Smo and Kif7 is absent in the other four Dlg proteins. Taking into consideration these structural differences, it seems unlikely that these other Dlg family members can compensate for loss of Dlg5 function. Interestingly, survival through gestation until birth is reported for mice lacking Evc2 (Caparrós-Martín et al. 2013), which may also function in the Dlg5-dependent Gli activation arm of $\mathrm{Hh}$ response (see above).

The major source of activated Gli transcriptional activity is Gli2, and in this regard, it is worth noting that embryos lacking Gli2 function survive to late stages of gestation with surprisingly minor patterning defects. The 
ability to generate Gli repressor primarily from proteolytic processing of Gli3 in a Hh-regulated manner thus suffices to supply a substantial proportion of the Hhmediated patterning activity required for embryonic development. It is worth noting in this context that $D l g 5^{-/-}$ animals display defects in lung branching (Nechiporuk et al. 2013) very much like those associated with genetic defects in Hh signaling (Bellusci et al. 1997; Pepicelli et al. 1998; Miller et al. 2004). In addition, Dlg5 single-nucleotide polymorphisms (SNPs) have been identified in numerous families of European descent with inflammatory bowel disease (IBD) (Friedrichs and Stoll 2006; Henckaerts et al. 2008), an intriguing observation in light of previous work implicating Hh response in IBD and the regulation of inflammatory pathways in the gut (Nielsen et al. 2004; Lees et al. 2008; Zacharias et al. 2010).

We note that localization of Dlg5 at the basal body places it at an early point in the Smo activity cycle. Further work will be required to determine how many other factors, possibly including the tripartite Evc2 complex, may be specifically dedicated to the Dlg5-dependent Gli activation arm of Hedgehog response. We also anticipate the possibility that specific factors, perhaps also localized at the base of the cilium, may be involved in directing Smo action to the suppression of Gli repressor formation via expulsion of Gpr161 from the primary cilium.

\section{Materials and methods}

\section{Antibodies, chemicals, and small molecules}

The following commercial antibodies were used: anti-acetylated tubulin (T6793, Sigma), anti- $\gamma$-tubulin (T6557, Sigma), anti-GFP (A-11122, Invitrogen), anti-Flag M2 (F3165, Sigma), anti- $\beta$-actin (C4) (sc-47778, Santa Cruz Biotechnology), anti-CK1 $\alpha$ (C19) (sc6477, Santa Cruz Biotechnology), anti-Gli1 (L42B10) (2643, Cell Signaling), and anti-Gli3 (AF3690, R\&D Systems). The following antibodies have been described elsewhere: anti-Smo (Kim et al. 2009), anti-Dlg5 (Nechiporuk et al. 2007), anti-Kif7 (Liem et al. 2012), anti-Gli2 (Cho et al. 2008), and anti-Gpr161 (Mukhopadhyay et al. 2013). For immunoblotting, horseradish peroxidase-conjugated goat anti-mouse and anti-rabbit (Promega) and donkey anti-goat (Jackson ImmunoResearch) secondary antibodies were used. For immunofluorescence microscopy, Alexa Fluor 488- and 594-conjugated secondary antibodies were from Life Technologies. SAG1.5 was purchased from Enzo Life Sciences, DDM and CHS were purchased from Affymetrix, and Cytochalasin B and nocodazole were purchased from Sigma. Cyclopamine was a generous gift from Dale Gardner (US Department of Agriculture). ShhN conditioned medium and ShhNp protein were prepared as previously described (Taipale et al. 2000).

\section{DNA constructs}

LAP-Smo was constructed by inserting the Smo signal sequence (residues 1-33) upstream of the GFP tag in the retroviral vector pBabe-Puro (Morgenstern and Land 1990) prior to the subcloning of Smo (lacking the signal sequence) downstream from the LAP cassette (GFP-TEV site-S peptide). The pCMVSport6-StrawberryDlg5 vector (Nechiporuk et al. 2007) was used as the template for the PCR of Dlg5. All C-terminal 3xFlag and EGFP constructs were made by the subcloning of the respective PCR products into the pCMV14-3xFlag (Sigma) and pEGFP-N3 (Clontech) vectors, respectively. Mutations were generated using the QuikChange Lightning site-directed mutagenesis kit (Agilent Technologies) per the manufacturer's instructions.

\section{Cell culture}

NIH3T3 cells were grown in Dulbecco's modified Eagle's medium (DMEM) supplemented with $10 \%$ bovine calf serum (BCS) (Hyclone) and $1 \%$ penicillin/streptomycin/glutamine (PSQ). $\mathrm{Smo}^{-1-}, \mathrm{Dlg}^{-/-}, \mathrm{Ptch}^{-1-}$, and $\mathrm{Sufu}^{-/-} \mathrm{MEFs}$ were grown in DMEM supplemented with $10 \%$ fetal bovine serum (FBS) (Omega Scientific) and 1\% PSQ. HEK293T and Phoenix ampho cells were grown in DMEM supplemented with 10\% FBS, $1 \mathrm{mM}$ sodium pyruvate (Life Technologies), and 1\% PSQ. For generation of retroviruses, Phoenix ampho packaging cells were transfected with retroviral vectors using calcium phosphate, and retroviral supernatant was collected $2 \mathrm{~d}$ after transfection. Cells were infected with the retrovirus via spin infection at $2500 \mathrm{rpm}$ for $90 \mathrm{~min}$ in the presence of $6 \mathrm{\mu g} / \mathrm{mL}$ polybrene (EMD). $\mathrm{Smo}^{-1-}$ MEFs stably expressing LAP-Smo were generated by infecting $\mathrm{Smo}^{-1-}$ cells with retroviral LAP-Smo, and single GFP-positive cells were sorted by flow cytometry (FACS). Dlg $5^{-/-}$MEFs stably expressing LAP-Dlg5 were similarly generated by infecting $D \lg 5^{-1-}$ cells with retroviral LAP-Dlg5. MEFs were reversetransfected with esiRNAs targeting EGFP or Dlg5 (\#EMU182641, Sigma) using Lipofectamine RNAiMax (Invitrogen) according to the manufacturer's instructions. For Gli luciferase assays, cells were transfected with a mixture of $8 x G$ Gi-Firefly-luciferase and SV40-Renilla plasmids using TransIT-2020 transfection reagent (Mirus). For testing the functionality of Smo mutants in $\mathrm{Smo}^{-/-}$ MEFs, Smo alleles (at $5 \%$ by mass) were cotransfected with the reporter plasmids. Upon confluence, cells were shifted to DMEM with $0.5 \%$ serum containing ShhN conditioned medium (1:10 dilution) or other small molecules as indicated and incubated for an additional $36 \mathrm{~h}$, after which luciferase activities were measured using the dual-luciferase reporter assay system (Promega) and a Berthold Centro XS3 luminometer.

\section{SILAC and LC-MS/MS analysis}

LAP-Smo cells were grown in L-lysine and L-arginine-deficient DMEM (Pierce) supplemented with $10 \%$ dialyzed FBS (Pierce) and PSQ for 2 wk. The "light" culture was additionally supplemented with unlabeled L-lysine and L-arginine (Cambridge Isotopes), whereas the "heavy" culture was supplemented with L-lysine-2HCL (U- ${ }^{13} \mathrm{C}_{6}, 99 \%$; U- ${ }^{15} \mathrm{~N}_{2}, 99 \%$ ) (Cambridge Isotopes) and L-arginine- $\mathrm{HCl}\left({ }^{13} \mathrm{C}_{6},{ }^{15} \mathrm{~N}_{4}\right)$ (Pierce). Upon confluence, cells were starved overnight with DMEM containing 0.5\% dialyzed FBS. The "light" culture was then stimulated with ShhNp and SAG1.5 for an additional $4 \mathrm{~h}$. Cultures were then lysed in a solubilization buffer $(50 \mathrm{mM}$ Tris at $\mathrm{pH} 7.4,20 \%$ glycerol, $100 \mathrm{mM} \mathrm{KCl}, 1 \mathrm{mM} \mathrm{MgCl} 2,2 \mathrm{mM} \mathrm{DTT}, 90 \mathrm{U} / \mathrm{mL}$ benzonase, $1 \%$ DDM, $0.1 \%$ CHS) supplemented with Halt protease and phosphatase inhibitors (Pierce). Precleared lysates from the "light" and "heavy" cultures were then incubated individually with anti-GFP microbeads (Miltenyi Biotech) for $2 \mathrm{~h}$ at $4^{\circ} \mathrm{C}$. The two suspensions were then transferred to separate $\mathrm{M}$ columns (Miltenyi Biotech) for isolation of bound proteins. In-column digestion of proteins was performed by direct incubation of column-trapped beads with trypsin (Promega) under reducing conditions (1 mM DTT, $2 \mathrm{M}$ urea) for $1 \mathrm{~h}$. Digested peptides were eluted in a buffer containing 5 $\mathrm{mM}$ iodoacetamide and $2 \mathrm{M}$ urea. Peptides from the "light" and "heavy" samples were combined and incubated overnight in the dark for the cysteinyl-selective alkylation of reduced peptides. Peptide identification and relative quantification were determined 
by LC-MS/MS. Desalted, tryptic peptides were resolved by microcapillary reversed phase chromatography (EASY-nLC II [Proxeon]; $75-\mu \mathrm{m}$ ID column with pulled-needle emitter [New Objective] custom packed with C18 Ultro 120 matrix [Peake Scientific], $25 \mathrm{~cm}$ in length) and subjected to precursor and fragmentation analyses on a LTQ Orbitrap Velos mass spectrometer (ThermoFisher Scientific). High-resolution $(60,000)$ precursor scans in the Orbitrap were followed by "top12" tandem MS analyses (CID or ETD; on-board data-dependent decision tree logic) in the LIT. Peptide/protein identifications and ratio calculations were performed using MaxQuant (Cox and Mann 2008). Data were searched against a mouse protein database using the built-in Andromeda search engine (Cox et al. 2011). Methionine oxidation and $\mathrm{N}$ terminus acetylation were set as variable modifications, whereas cysteine carbamidomethylation was set as a fixed modification. Peptide and protein false discovery rates were fixed at 0.01 .

\section{Immunoprecipitation and immunoblotting}

HEK293T cells were transfected with the indicated constructs using FuGENE 6 (Promega). Whole-cell lysates were incubated with GFP-Trap magnetic beads (Chromotek) for immunoprecipitation of GFP-tagged proteins. For cross-linking of proteins in $L A P$ Smo2 MEFs, cells were incubated with $1 \mathrm{mM}$ DSP (Pierce) for $2 \mathrm{~h}$ at $4^{\circ} \mathrm{C}$. The reaction was then quenched $(100 \mathrm{mM}$ Tris at $\mathrm{pH} 7.4$ for 30 min at $4^{\circ} \mathrm{C}$ ), and cells were lysed in a DTT-free solubilization buffer $50 \mathrm{mM}$ Tris at $\mathrm{pH} 7.4,20 \%$ glycerol, $100 \mathrm{mM} \mathrm{KCl}, 1 \mathrm{mM} \mathrm{MgCl}$, $1 \%$ DDM, $0.1 \%$ CHS) supplemented with Halt protease and phosphatase inhibitors. GFP-Trap magnetic beads were used for immunoprecipitation, and bound proteins were eluted in SDS sample buffer containing $50 \mathrm{mM}$ DTT. Bands on Western blots were quantified using ImageJ software (National Institutes of Health) or Image Lab (Bio-Rad) software.

\section{Immunofluorescence}

Cells were grown to confluency on glass coverslips in 12-well plates and then starved overnight in DMEM containing $0.5 \%$ serum with the indicated treatments. Cells were fixed in either $4 \%$ paraformaldehyde for $20 \mathrm{~min}$ at room temperature or methanol for $5 \mathrm{~min}$ at $-20^{\circ} \mathrm{C}$ (for staining of Smo or $\gamma$-tubulin). After blocking in $1.5 \%$ normal goat serum (Jackson ImmunoResearch), fixed cells were incubated with the indicated primary and secondary antibodies and mounted using ProLong Gold anti-fade reagent with DAPI (Life Technologies). Images were taken on a Leica SD6000 spinning-disc confocal microscope equipped with an EM-CCD (electron-multiplying charge-coupled device) camera and a Z-galvanometer stage and processed using LAS AF software (Leica). All wide-field Z-stack images were deconvoluted through three blind iterative cycles. The amount of Kif7 and Gli2 at the ciliary tip or in the nucleus was quantified using ImageJ software. Each RGB image was first split into three channel images. Using the acetylated tubulin (red) or DAPI (blue) channel, a threshold value was selected, and the image was converted into a binary image. A mask was then constructed at the tip of each primary cilium or enclosing the nucleus in a given image. All masked regions from the acetylated tubulin or DAPI channel were copied and pasted into the Kif7/Gli2 channel (green channel), and the fluorescence intensities were measured.

\section{Centrosome fractionation}

Centrosomes were isolated using a discontinuous sucrose gradient centrifugation method (Meigs and Kaplan 2008; Reber 2011). Upon reaching confluency, cells were treated with $5 \mu \mathrm{g} / \mathrm{mL}$ Cytochalasin $\mathrm{B}$ and nocodazole for $1 \mathrm{~h}$ at $37^{\circ} \mathrm{C}$ to induce the depolymerization of cytoplasmic actin filaments and microtubules, respectively. Cells were washed with HB buffer $(20 \mathrm{mM}$ Hepes at $\mathrm{pH} 7.8,5 \mathrm{mM}$ potassium acetate, $0.5 \mathrm{mM} \mathrm{MgCl}_{2}, 0.5$ $\mathrm{mM}$ DTT) and then incubated for $10 \mathrm{~min}$ at $4^{\circ} \mathrm{C}$ with $\mathrm{HB}$ buffer supplemented with protease inhibitors (Roche). After aspiration, cells were scraped into residual $\mathrm{HB}$ buffer and then Dounce homogenized with 10 strokes to disrupt the cell membrane. Cell debris and chromatin were pelleted by centrifuging the lysate at $3000 \mathrm{rpm}$ for $5 \mathrm{~min}$, and Triton X-100 was then added to the supernatant to a final concentration of $0.1 \%$. The supernatant was loaded onto a step sucrose gradient $170 \%, 50 \%$, and $40 \%[\mathrm{w} / \mathrm{w}]$ sucrose) and centrifuged at $26,000 \mathrm{rpm}$ in a SW28Ti rotor for $1 \mathrm{~h}$ at $4^{\circ} \mathrm{C}$. Next, $0.5 \mathrm{~mL}$ fractions were removed, and aliquots were analyzed by SDS-PAGE and immunoblotting for $\gamma$-tubulin-enriched fractions.

\section{Acknowledgments}

We thank V. Vasioukhin for his generous gifts of Dlg5 antibodies and Dlg5 $5^{+/-}$and Dlg5 $5^{-1-}$ MEFs. We also thank K. Anderson, J. Eggenschwiler, and P. Jackson for their generous gifts of Kif7, Gli2, and Gpr161 antibodies, respectively, and members of the P.A.B. laboratory for valuable input. Y.C.C. is a fellow of the Agency for Science, Technology, and Research (A*STAR). P.A.B. is an investigator of the Howard Hughes Medical Institute. This work was supported by National Institutes of Health National Institute of General Medical Sciences grant R01GM102498 and the Howard Hughes Medical Institute.

\section{References}

Beachy PA, Karhadkar SS, Berman DM. 2004. Tissue repair and stem cell renewal in carcinogenesis. Nature 432: 324-331.

Bellusci S, Furuta Y, Rush MG, Henderson R, Winnier G, Hogan BL. 1997. Involvement of Sonic hedgehog (Shh) in mouse embryonic lung growth and morphogenesis. Development 124: 53-63.

Breslow DK, Koslover EF, Seydel F, Spakowitz AJ, Nachury MV. 2013. An in vitro assay for entry into cilia reveals unique properties of the soluble diffusion barrier. I Cell Biol 203: 129-147.

Caparrós-Martín JA, Valencia M, Reytor E, Pacheco M, Fernandez M, Perez-Aytes A, Gean E, Lapunzina P, Peters H, Goodship JA, et al. 2013. The ciliary Evc/Evc2 complex interacts with Smo and controls Hedgehog pathway activity in chondrocytes by regulating Sufu/Gli3 dissociation and Gli3 trafficking in primary cilia. Hum Mol Genet 22: 124-139.

Chen MH, Wilson CW, Li YJ, Law KK, Lu CS, Gacayan R, Zhang X, Hui CC, Chuang PT. 2009. Cilium-independent regulation of Gli protein function by Sufu in Hedgehog signaling is evolutionarily conserved. Genes Dev 23: 19101928.

Cheung HO, Zhang X, Ribeiro A, Mo R, Makino S, Puviindran V, Law KK, Briscoe J, Hui CC. 2009. The kinesin protein Kif7 is a critical regulator of Gli transcription factors in mammalian hedgehog signaling. Sci Signal 2: ra29.

Cho A, Ko HW, Eggenschwiler JT. 2008. FKBP8 cell-autonomously controls neural tube patterning through a Gli2- and Kif3a-dependent mechanism. Dev Biol 321: 27-39.

Cohen C, Parry DA. 1990. $\alpha$-Helical coiled coils and bundles: how to design an $\alpha$-helical protein. Proteins 7: 1-15.

Corbit KC, Aanstad P, Singla V, Norman AR, Stainier DY, Reiter JF. 2005. Vertebrate Smoothened functions at the primary cilium. Nature 437: 1018-1021.

Cox J, Mann M. 2008. MaxQuant enables high peptide identification rates, individualized p.p.b.-range mass accuracies 
and proteome-wide protein quantification. Nat Biotechnol 26: $1367-1372$.

Cox J, Neuhauser N, Michalski A, Scheltema RA, Olsen JV, Mann M. 2011. Andromeda: a peptide search engine integrated into the MaxQuant environment. I Proteome Res 10: $1794-1805$.

Dominguez M, Brunner M, Hafen E, Basler K. 1996. Sending and receiving the hedgehog signal: control by the Drosophila Gli protein Cubitus interruptus. Science 272: 1621-1625.

Endoh-Yamagami S, Evangelista M, Wilson D, Wen X, Theunissen JW, Phamluong K, Davis M, Scales SJ, Solloway MJ, de Sauvage FJ, et al. 2009. The mammalian Cos2 homolog Kif7 plays an essential role in modulating Hh signal transduction during development. Curr Biol 19: 1320-1326.

Evangelista M, Lim TY, Lee J, Parker L, Ashique A, Peterson AS, Ye W, Davis DP, de Sauvage FJ. 2008. Kinome siRNA screen identifies regulators of ciliogenesis and hedgehog signal transduction. Sci Signal 1: ra7.

Friedrichs F, Stoll M. 2006. Role of discs large homolog 5. World J Gastroenterol 12: 3651-3656.

He M, Subramanian R, Bangs F, Omelchenko T, Liem KF Jr, Kapoor TM, Anderson KV. 2014. The kinesin-4 protein Kif7 regulates mammalian Hedgehog signalling by organizing the cilium tip compartment. Nat Cell Biol 16: 663-672.

Henckaerts L, Figueroa C, Vermeire S, Sans M. 2008. The role of genetics in inflammatory bowel disease. Curr Drug Targets 9: $361-368$.

Hillman RT, Feng BY, Ni J, Woo WM, Milenkovic L, Hayden Gephart MG, Teruel MN, Oro AE, Chen JK, Scott MP. 2011. Neuropilins are positive regulators of Hedgehog signal transduction. Genes Dev 25: 2333-2346.

Hubner NC, Bird AW, Cox J, Splettstoesser B, Bandilla P, Poser I, Hyman A, Mann M. 2010. Quantitative proteomics combined with BAC TransgeneOmics reveals in vivo protein interactions. J Cell Biol 189: 739-754.

Hung AY, Sheng M. 2002. PDZ domains: structural modules for protein complex assembly. J Biol Chem 277: 5699-5702.

Ingham PW, Nakano Y, Seger C. 2011. Mechanisms and functions of Hedgehog signalling across the metazoa. Nat Rev Genet 12: 393-406.

Jacob LS, Wu X, Dodge ME, Fan CW, Kulak O, Chen B, Tang W, Wang B, Amatruda JF, Lum L. 2011. Genome-wide RNAi screen reveals disease-associated genes that are common to Hedgehog and Wnt signaling. Sci Signal 4: ra4.

Jakobsen L, Vanselow K, Skogs M, Toyoda Y, Lundberg E, Poser I, Falkenby LG, Bennetzen M, Westendorf J, Nigg EA, et al. 2011. Novel asymmetrically localizing components of human centrosomes identified by complementary proteomics methods. EMBO J 30: 1520-1535.

Katoh Y, Katoh M. 2004. Characterization of KIF7 gene in silico. Int J Oncol 25: 1881-1886.

Kim J, Kato M, Beachy PA. 2009. Gli2 trafficking links Hedgehog-dependent activation of Smoothened in the primary cilium to transcriptional activation in the nucleus. Proc Natl Acad Sci 106: 21666-21671.

Klejnot M, Kozielski F. 2012. Structural insights into human Kif7, a kinesin involved in Hedgehog signalling. Acta Crystallogr D Biol Crystallogr 68: 154-159.

Lees CW, Zacharias WJ, Tremelling M, Noble CL, Nimmo ER, Tenesa A, Cornelius J, Torkvist L, Kao J, Farrington S, et al. 2008. Analysis of germline GLI1 variation implicates hedgehog signalling in the regulation of intestinal inflammatory pathways. PLoS Med 5: e239.

Liem KF Jr, He M, Ocbina PJ, Anderson KV. 2009. Mouse Kif7/ Costal2 is a cilia-associated protein that regulates Sonic hedgehog signaling. Proc Natl Acad Sci 106: 13377-13382.
Liem KF Jr, Ashe A, He M, Satir P, Moran J, Beier D, Wicking C, Anderson KV. 2012. The IFT-A complex regulates Shh signaling through cilia structure and membrane protein trafficking. J Cell Biol 197: 789-800.

Lin YC, Niewiadomski P, Lin B, Nakamura H, Phua SC, Jiao J, Levchenko A, Inoue T, Rohatgi R. 2013. Chemically inducible diffusion trap at cilia reveals molecular sieve-like barrier. Nat Chem Biol 9: 437-443.

Lum L, Zhang C, Oh S, Mann RK, von Kessler DP, Taipale J, Weis-Garcia F, Gong R, Wang B, Beachy PA. 2003. Hedgehog signal transduction via Smoothened association with a cytoplasmic complex scaffolded by the atypical kinesin, Costal-2. Mol Cell 12: 1261-1274.

Maurya AK, Ben J, Zhao Z, Lee RT, Niah W, Ng AS, Iyu A, Yu W, Elworthy S, van Eeden FJ, et al. 2013. Positive and negative regulation of Gli activity by Kif7 in the zebrafish embryo. PLoS Genet 9: e1003955.

Mayer BJ. 2001. SH3 domains: complexity in moderation. J Cell Sci 114: 1253-1263.

Meigs TE, Kaplan DD. 2008. Isolation of centrosomes from cultured mammalian cells. Cold Spring Harbor Protoc doi: 10.1101/pdb.prot5039.

Merchant M, Evangelista M, Luoh SM, Frantz GD, Chalasani S, Carano RA, van Hoy M, Ramirez J, Ogasawara AK, McFarland LM, et al. 2005. Loss of the serine/threonine kinase fused results in postnatal growth defects and lethality due to progressive hydrocephalus. Mol Cell Biol 25: 7054-7068.

Methot N, Basler K. 1999. Hedgehog controls limb development by regulating the activities of distinct transcriptional activator and repressor forms of Cubitus interruptus. Cell 96: 819-831.

Miller LA, Wert SE, Clark JC, Xu Y, Perl AK, Whitsett JA. 2004. Role of Sonic hedgehog in patterning of tracheal-bronchial cartilage and the peripheral lung. Dev Dyn 231: 57-71.

Morgenstern JP, Land H. 1990. A series of mammalian expression vectors and characterisation of their expression of a reporter gene in stably and transiently transfected cells. Nucleic Acids Res 18: 1068.

Mukhopadhyay S, Wen X, Ratti N, Loktev A, Rangell L, Scales SJ, Jackson PK. 2013. The ciliary G-protein-coupled receptor Gpr161 negatively regulates the Sonic hedgehog pathway via cAMP signaling. Cell 152: 210-223.

Nakamura H, Sudo T, Tsuiki H, Miyake H, Morisaki T, Sasaki J, Masuko N, Kochi M, Ushio Y, Saya H. 1998. Identification of a novel human homolog of the Drosophila dlg, P-dlg, specifically expressed in the gland tissues and interacting with p55. FEBS Lett 433: 63-67.

Nechiporuk T, Fernandez TE, Vasioukhin V. 2007. Failure of epithelial tube maintenance causes hydrocephalus and renal cysts in Dlg5 $5^{-1-}$ mice. Dev Cell 13: 338-350.

Nechiporuk T, Klezovitch O, Nguyen L, Vasioukhin V. 2013. Dlg5 maintains apical aPKC and regulates progenitor differentiation during lung morphogenesis. Dev Biol 377: 375-384.

Nielsen CM, Williams J, van den Brink GR, Lauwers GY, Roberts DJ. 2004. Hh pathway expression in human gut tissues and in inflammatory gut diseases. Lab Invest 84: 1631-1642.

Ong SE, Blagoev B, Kratchmarova I, Kristensen DB, Steen H, Pandey A, Mann M. 2002. Stable isotope labeling by amino acids in cell culture, SILAC, as a simple and accurate approach to expression proteomics. Mol Cell Proteomics 1: 376-386.

Pan Y, Bai CB, Joyner AL, Wang B. 2006. Sonic hedgehog signaling regulates Gli2 transcriptional activity by suppressing its processing and degradation. Mol Cell Biol 26: 3365-3377. 
Chong et al.

Pepicelli CV, Lewis PM, McMahon AP. 1998. Sonic hedgehog regulates branching morphogenesis in the mammalian lung. Curr Biol 8: 1083-1086.

Purmonen S, Ahola TM, Pennanen P, Aksenov N, Zhuang YH, Tuohimaa P, Ylikomi T. 2002. HDLG5/KIAA0583, encoding a MAGUK-family protein, is a primary progesterone target gene in breast cancer cells. Int I Cancer 102: 1-6.

Pusapati GV, Hughes CE, Dorn KV, Zhang D, Sugianto P, Aravind L, Rohatgi R. 2014. EFCAB7 and IQCE regulate hedgehog signaling by tethering the EVC-EVC2 complex to the base of primary cilia. Dev Cell 28: 483-496.

Reber S. 2011. Isolation of centrosomes from cultured cells. Methods Mol Biol 777: 107-116.

Robbins DJ, Nybakken KE, Kobayashi R, Sisson JC, Bishop JM, Therond PP. 1997. Hedgehog elicits signal transduction by means of a large complex containing the kinesin-related protein costal2. Cell 90: 225-234.

Rohatgi R, Milenkovic L, Scott MP. 2007. Patched1 regulates hedgehog signaling at the primary cilium. Science 317: 372-376.

Shah G, Brugada R, Gonzalez O, Czernuszewicz G, Gibbs RA, Bachinski L, Roberts R. 2002. The cloning, genomic organization and tissue expression profile of the human DLG5 gene. BMC Genomics 3: 14.

Sisson JC, Ho KS, Suyama K, Scott MP. 1997. Costal2, a novel kinesin-related protein in the Hedgehog signaling pathway. Cell 90: 235-245.

Sparks AB, Rider JE, Hoffman NG, Fowlkes DM, Quillam LA, Kay BK. 1996. Distinct ligand preferences of Src homology 3 domains from Src, Yes, Abl, Cortactin, p53bp2, PLC $\gamma$, Crk, and Grb2. Proc Natl Acad Sci 93: 1540-1544.

Sparks AB, Rider JE, Kay BK. 1998. Mapping the specificity of SH3 domains with phage-displayed random-peptide libraries. Methods Mol Biol 84: 87-103.

Stegman MA, Vallance JE, Elangovan G, Sosinski J, Cheng Y, Robbins DJ. 2000. Identification of a tetrameric hedgehog signaling complex. J Biol Chem 275: 21809-21812.

Taipale J, Chen JK, Cooper MK, Wang B, Mann RK, Milenkovic L, Scott MP, Beachy PA. 2000. Effects of oncogenic mutations in Smoothened and Patched can be reversed by cyclopamine. Nature 406: 1005-1009.

Teglund S, Toftgard R. 2010. Hedgehog beyond medulloblastoma and basal cell carcinoma. Biochim Biophys Acta 1805: 181-208.

Tuson M, He M, Anderson KV. 2011. Protein kinase A acts at the basal body of the primary cilium to prevent Gli2 activation and ventralization of the mouse neural tube. Development 138: 4921-4930.

Varjosalo M, Taipale J. 2008. Hedgehog: functions and mechanisms. Genes Dev 22: 2454-2472.

Wang QT, Holmgren RA. 2000. Nuclear import of Cubitus interruptus is regulated by hedgehog via a mechanism distinct from Ci stabilization and Ci activation. Development 127: 3131-3139.

Wang B, Fallon JF, Beachy PA. 2000. Hedgehog-regulated processing of Gli3 produces an anterior/posterior repressor gradient in the developing vertebrate limb. Cell 100: 423-434.

Wilson CW, Nguyen CT, Chen MH, Yang JH, Gacayan R, Huang J, Chen JN, Chuang PT. 2009. Fused has evolved divergent roles in vertebrate Hedgehog signalling and motile ciliogenesis. Nature 459: 98-102.

Woods DF, Bryant PJ. 1991. The discs-large tumor suppressor gene of Drosophila encodes a guanylate kinase homolog localized at septate junctions. Cell 66: 451-464.

Woods DF, Hough C, Peel D, Callaini G, Bryant PJ. 1996. Dlg protein is required for junction structure, cell polarity, and proliferation control in Drosophila epithelia. J Cell Biol 134: 1469-1482.

Yang C, Chen W, Chen Y, Jiang J. 2012. Smoothened transduces Hedgehog signal by forming a complex with Evc/Evc2. Cell Res 22: 1593-1604.

Zacharias WJ, Li X, Madison BB, Kretovich K, Kao JY, Merchant JL, Gumucio DL. 2010. Hedgehog is an anti-inflammatory epithelial signal for the intestinal lamina propria. Gastroenterology 138: 2368-2377.e4.

Zhu H, Pan S, Gu S, Bradbury EM, Chen X. 2002. Amino acid residue specific stable isotope labeling for quantitative proteomics. Rapid Commun Mass Spectrom 16: 2115-2123. 


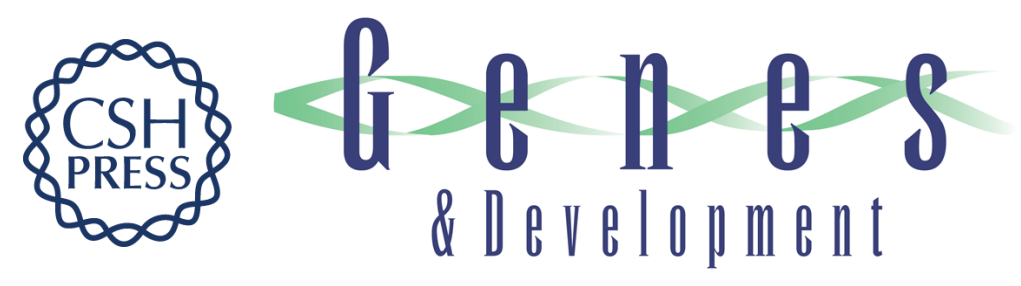

\section{Bifurcating action of Smoothened in Hedgehog signaling is mediated by Dlg5}

Yong Chun Chong, Randall K. Mann, Chen Zhao, et al.

Genes Dev. 2015, 29:

Access the most recent version at doi:10.1101/gad.252676.114

\section{Supplemental http://genesdev.cshlp.org/content/suppl/2015/02/02/29.3.262.DC1 Material}

References This article cites 68 articles, 26 of which can be accessed free at: http://genesdev.cshlp.org/content/29/3/262.full.html\#ref-list-1

Creative This article is distributed exclusively by Cold Spring Harbor Laboratory Press for the first Commons six months after the full-issue publication date (see

License http://genesdev.cshlp.org/site/misc/terms.xhtml). After six months, it is available under a Creative Commons License (Attribution-NonCommercial 4.0 International), as described at http://creativecommons.org/licenses/by-nc/4.0/.

Email Alerting Receive free email alerts when new articles cite this article - sign up in the box at the top Service right corner of the article or click here.

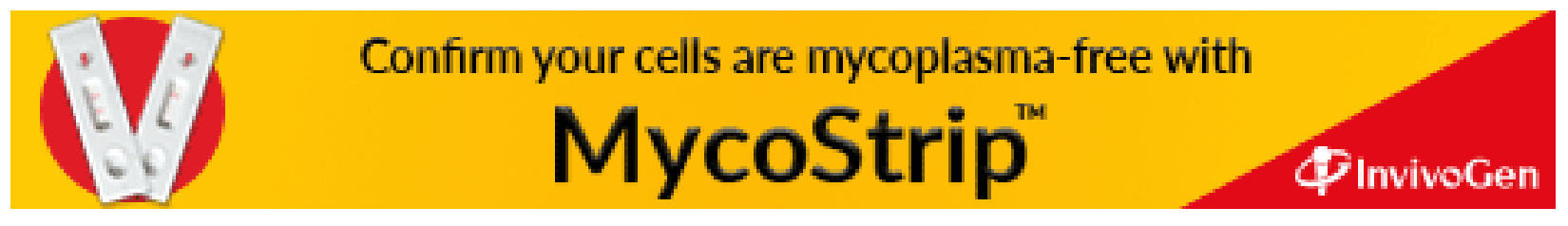

